\title{
A New Efficient Catalytic System for the Chemoselective Cobalt-Catalyzed Cross-Coupling of Aryl Grignard Reagents with Primary and Secondary Alkyl Bromides
}

Gérard Cahiez, Christophe Chaboche, Christophe Duplais and Alban Moyeux

\author{
Department of Chemistry \\ CNRS - Université de Paris 13 \\ 74 rue Marcel Cachin \\ F-93017 Bobigny, France \\ *gerard.cahiez@univ-paris13.fr
}

\section{Supporting Information}

\section{Summary}

1- General....

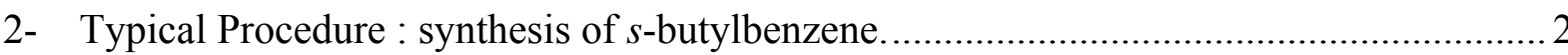

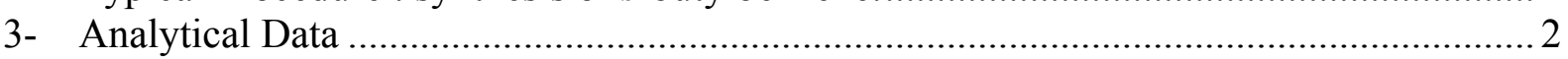

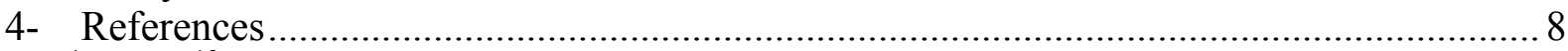

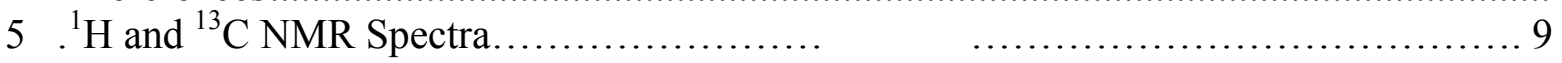

\section{1- General}


All reactions were carried out under a nitrogen atmosphere. All starting materials were purchased from commercial sources and used without any further purification. Anhydrous THF was purchased from commercial source. Yields refer to isolated yields of compounds estimated to be $\geq 97 \%$ pure as determined by ${ }^{1} \mathrm{H}-\mathrm{NMR}(400 \mathrm{MHz}),{ }^{13} \mathrm{C}-\mathrm{NMR}(100 \mathrm{MHz})$ and GC analysis (capillary column $30 \mathrm{~m} \times 0.25 \mathrm{~mm} \times 0.25 \mu \mathrm{m}$ ). All compounds give satisfactory centesimal analysis. The analytical data for the known compounds were found to match with the literature data.

Ionization for High Resolution Mass Spectra (HRMS) was obtained by electronic impact (EI, $70 \mathrm{eV}$ ). Mass spectra are reported as $\mathrm{m} / \mathrm{z}$.

The Grignard solutions were titrated according to the procedure described by Watson ${ }^{1}$.

\section{2- Typical Procedure : synthesis of s-butylbenzene (3d).}

A dry and nitrogen flushed $250 \mathrm{~mL}$ four-necked flask, equipped with a mechanical stirrer, a thermometer, a nitrogen inlet and a septum, was charged with THF $(25 \mathrm{~mL}), 2$-bromobutane (6.85 g, $50 \mathrm{mmol}), \mathrm{CoCl}_{2}(325 \mathrm{mg}, 5 \mathrm{~mol} \%)$ and TMEDA (0.29 g, $\left.5 \mathrm{~mol} \%\right)$. The reaction mixture was cooled to $0^{\circ} \mathrm{C}$ then a solution of phenylmagnesium bromide in THF $(61 \mathrm{~mL}, 0.9$ M, $55 \mathrm{mmol}$ ) was added in $50 \mathrm{~min}$. After completion of the addition, the reaction mixture was stirred for $30 \mathrm{~min}$ then quenched with aqueous $\mathrm{HCl} 1 \mathrm{M}(100 \mathrm{~mL})$. The aqueous layer was extracted with ether $(3 \times 50 \mathrm{~mL})$, then the combined organic layers were dried with $\mathrm{MgSO}_{4}$ and concentrated in vacuo. The crude product is then distillated under reduced pressure (55 ${ }^{\circ} \mathrm{C}, 10$ Torr) to yield $6.31 \mathrm{~g}(94 \%)$ of s-butylbenzene as a colorless oil.

\section{3- Analytical Data}

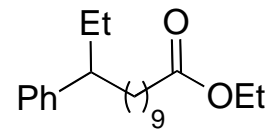

2

${ }^{1} \mathbf{H}\left(\mathbf{4 0 0 M H z}, \mathbf{C D C l}_{3}\right) \delta(\mathbf{p p m}): 0.69(\mathrm{t}, \mathrm{J}=7.33 \mathrm{~Hz}, 3 \mathrm{H}), 1-1.3(\mathrm{~m}, 13 \mathrm{H}), 1.49(\mathrm{~m}, 6 \mathrm{H}), 2.19$ $(\mathrm{t}, \mathrm{J}=7.33 \mathrm{~Hz}, 2 \mathrm{H}), 2.30(\mathrm{~m}, 1 \mathrm{H}), 4.03(\mathrm{q}, \mathrm{J}=7.33 \mathrm{~Hz}, 2 \mathrm{H}), 7.11(\mathrm{~d}, \mathrm{~J}=7.33 \mathrm{~Hz}, 2 \mathrm{H}), 7.16$ (t, J = 7.33 Hz, 1H), $7.27(\mathrm{t}, \mathrm{J}=7.33 \mathrm{~Hz}, 2 \mathrm{H})$. 
${ }^{13} \mathbf{C}\left(\mathbf{1 0 0 M H z}, \mathbf{C D C l}_{3}\right) \delta(\mathbf{p p m})$ : 12.18, 14.23, 24.93, 27.55, 29.08, 29.30, 29.41, 29.64, $29.75,29.69,29.80,34.47,36.61,47.84,60.11,125.68,127.70$ (2C), 128.09 (2C), 146.03, 174.01 .

Micro-analysis : calcd (\%) : $\mathrm{C}=78.90, \mathrm{H}=10.59, \mathrm{O}=10.51$

found $(\%): \mathrm{C}=79.17, \mathrm{H}=10.48, \mathrm{O}=10.35$

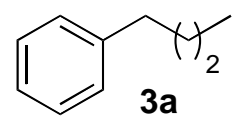

${ }^{1} \mathbf{H}\left(\mathbf{4 0 0 M H z}, \mathbf{C D C l}_{3}\right) \delta(\mathbf{p p m}): 0,96(\mathrm{t}, 7.33 \mathrm{~Hz}, 3 \mathrm{H}), 1,33(\mathrm{~m}, 2 \mathrm{H}), 1,65(\mathrm{~m}, 2 \mathrm{H}), 2,57(\mathrm{t}, \mathrm{J}=$ $7.33 \mathrm{~Hz}, 2 \mathrm{H}), 7,18(\mathrm{~d}, \mathrm{~J}=7.79 \mathrm{~Hz}, 2 \mathrm{H}), 7,21$ (t, J = 7.79 Hz,1H), 8,09 (t, J = 7.79 Hz, 2H).

${ }^{13} \mathbf{C}\left(\mathbf{1 0 0 M H z}, \mathbf{C D C l}_{3}\right) \delta(\mathbf{p p m})$ : 24.6, 27.4, 34.1, 38.2, 128.7, 130.4 (2C), 132.8 (2C), 138.0.

Micro-analysis : calcd (\%) : $\mathrm{C}=89.49, \mathrm{H}=10.51$ found $(\%): \mathrm{C}=89.61, \mathrm{H}=10.39$

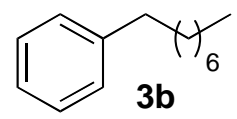

${ }^{1} \mathbf{H}\left(\mathbf{4 0 0 M H z}, \mathbf{C D C l}_{3}\right) \delta(\mathbf{p p m}): 0.88(\mathrm{t}, \mathrm{J}=6.87 \mathrm{~Hz}, 3 \mathrm{H}), 1.27(\mathrm{~m}, 10 \mathrm{H}), 1.60(\mathrm{~m}, 2 \mathrm{H}), 2.61$ $(\mathrm{t}, \mathrm{J}=7.79 \mathrm{~Hz}, 2 \mathrm{H}), 7.16(\mathrm{~m}, 3 \mathrm{H}), 7.26(\mathrm{~m}, 2 \mathrm{H})$.

${ }^{13} \mathbf{C}\left(\mathbf{1 0 0 M H z}, \mathbf{C D C l}_{3}\right) \delta(\mathbf{p p m})$ : 14.12, 22.68, 29.28, 29.36, 29.50, 31.58, 31.89, 35.99, $125.50,128.18(2 \mathrm{C}), 128.37$ (2C), 142.93.

Micro-analysis : calcd (\%) : $\mathrm{C}=88.35, \mathrm{H}=11.65$

found $(\%): \mathrm{C}=88.65, \mathrm{H}=11.35$

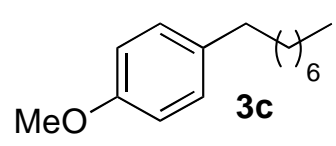

${ }^{1} \mathbf{H}\left(\mathbf{4 0 0 M H z}, \mathbf{C D C l}_{3}\right) \delta(\mathbf{p p m}): 0.87(\mathrm{t}, \mathrm{J}=6.87 \mathrm{~Hz}, 3 \mathrm{H}), 1.28(\mathrm{~m}, 10 \mathrm{H}), 1.58$ (quint. , J = $7.79 \mathrm{~Hz}, 2 \mathrm{H}), 2.53$ (t, J = 7.79 Hz, 2H), 3.79 (s, 3H), 6.82 (d, J = 8.70 Hz, 2H), 7.09 (d, J = $8.70 \mathrm{~Hz}, 2 \mathrm{H})$.

${ }^{13} \mathbf{C}\left(\mathbf{1 0 0 M H z}, \mathbf{C D C l}_{3}\right)$ S(ppm) : 14.12, 22.67, 29.28 (2C), 29.49, 31.80, 31.88, 35.02, 55.17, 113.54 (2C), 129.20 (2C), $135.01,157.47$. 
Micro-analysis : calcd (\%) : $\mathrm{C}=81.76, \mathrm{H}=10.98, \mathrm{O}=7.26$

found $(\%): \mathrm{C}=81.64, \mathrm{H}=11.12, \mathrm{O}=7.24$

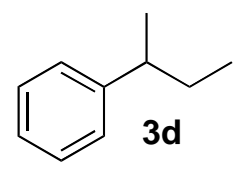

${ }^{1} \mathbf{H}\left(\mathbf{4 0 0 M H z}, \mathbf{C D C l}_{3}\right) \delta(\mathbf{p p m}): 0.83(\mathrm{t}, \mathrm{J}=7.33 \mathrm{~Hz}, 3 \mathrm{H}), 1.24(\mathrm{~d}, \mathrm{~J}=6.87 \mathrm{~Hz}, 3 \mathrm{H}), 1.59$ (quint. , $\mathrm{J}=7.33 \mathrm{~Hz}, 2 \mathrm{H}), 2.58(\mathrm{~s}, \mathrm{~J}=7.33 \mathrm{~Hz}, 1 \mathrm{H}), 7.17(\mathrm{~m}, 3 \mathrm{H}), 7.28(\mathrm{~d}, \mathrm{~J}=7.33 \mathrm{~Hz}, 2 \mathrm{H})$.

${ }^{13} \mathbf{C}\left(\mathbf{1 0 0 M H z}, \mathbf{C D C l}_{3}\right) \delta(\mathbf{p p m}): 12.27,21.85,31.16,41.65,125.73$ (2C), 127.03 (2C), 128.20, 147.63.

Micro-analysis : calcd (\%) : $\mathrm{C}=89.49, \mathrm{H}=10.51$

found $(\%): \mathrm{C}=89.32, \mathrm{H}=10.68$

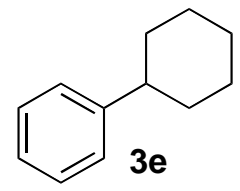

${ }^{1} \mathbf{H}\left(\mathbf{4 0 0 M H z}, \mathbf{C D C l}_{3}\right) \delta(\mathbf{p p m}): 1.25(\mathrm{~m}, 1 \mathrm{H}), 1.42(\mathrm{~m}, 4 \mathrm{H}), 1.75(\mathrm{~m}, 1 \mathrm{H}), 1.84(\mathrm{~m}, 4 \mathrm{H}), 2.49$ (m, 1H), $7.20(\mathrm{~m}, 3 \mathrm{H}), 7.27$ (m, 2H).

${ }^{13} \mathbf{C}\left(\mathbf{1 0 0 M H z}, \mathbf{C D C l}_{3}\right) \delta(\mathbf{p p m}): 26.14$ (2C), 26.89, 34.43 (2C), 44.56, 125.79 (2C), 126.79 (2C), 128.24, 148.05 .

Micro-analysis : calcd (\%) : $\mathrm{C}=89.94, \mathrm{H}=10.06$ found (\%) : $\mathrm{C}=89.86, \mathrm{H}=10.14$

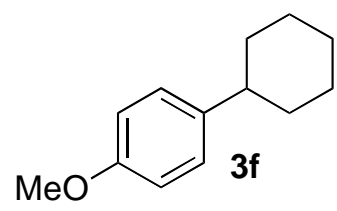

${ }^{1} \mathbf{H}\left(\mathbf{4 0 0 M H z}, \mathbf{C D C l}_{3}\right) \delta(\mathbf{p p m}): 1.23(\mathrm{~m}, 1 \mathrm{H}), 1.37$ (quint. , J = 8.70 Hz, 4H), $1.75(\mathrm{~m}, 1 \mathrm{H})$, $1.83(\mathrm{~m}, 4 \mathrm{H}), 2.44(\mathrm{~m}, 1 \mathrm{H}), 3.78(\mathrm{~s}, 3 \mathrm{H}), 6.83(\mathrm{~d}, \mathrm{~J}=9.62 \mathrm{~Hz}, 2 \mathrm{H}), 7.13$ (d, J = 9.62 Hz, 2H). ${ }^{13} \mathbf{C}\left(\mathbf{1 0 0 M H z}, \mathbf{C D C l}_{3}\right) \delta(\mathbf{p p m}):$ 26.13, 26.91(2C), 34.69 (2C), 43.64, 55.20, 113.57 (2C), 127.60 (2C), 140.34, 157.56.

Micro-analysis : calcd (\%) : $\mathrm{C}=82.06, \mathrm{H}=9.53, \mathrm{O}=8.41$ found $(\%): \mathrm{C}=81.99, \mathrm{H}=9.66, \mathrm{O}=8.35$ 


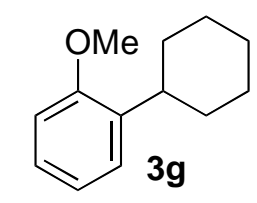

${ }^{1} \mathbf{H}\left(\mathbf{4 0 0 M H z}, \mathbf{C D C l}_{3}\right) \boldsymbol{\delta}(\mathbf{p p m}): 1.25(\mathrm{~m}, 1 \mathrm{H}), 1.38(\mathrm{~m}, 4 \mathrm{H}), 1.75(\mathrm{~m}, 1 \mathrm{H}), 1.83(\mathrm{~m}, 4 \mathrm{H}), 2.95$ $(\mathrm{m}, 1 \mathrm{H}), 3.80(\mathrm{~s}, 3 \mathrm{H}), 6.83(\mathrm{~d}, \mathrm{~J}=7.79 \mathrm{~Hz}, 1 \mathrm{H}), 6.91(\mathrm{t}, \mathrm{J}=7.33 \mathrm{~Hz}, 1 \mathrm{H}), 7.16(\mathrm{~d}, \mathrm{~J}=7.33$ $\mathrm{Hz}, 1 \mathrm{H}), 7.18$ (t, J = 7.79 Hz, 1H).

${ }^{13} \mathbf{C}\left(\mathbf{1 0 0 M H z}, \mathbf{C D C l}_{3}\right) \delta(\mathbf{p p m})$ : 26.41 (2C), 27.07, 33.17, 36.64 (2C), 55.29, 110.21, $120.47,126.41,126.46,136.15,156.60$.

Micro-analysis : $\operatorname{calcd}(\%): \mathrm{C}=82.06, \mathrm{H}=9.53, \mathrm{O}=8.41$ found $(\%): \mathrm{C}=81.98, \mathrm{H}=9.69, \mathrm{O}=8.33$

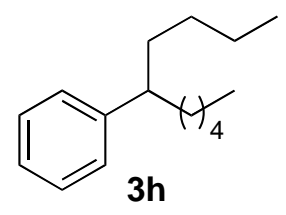

${ }^{1} \mathbf{H}\left(\mathbf{4 0 0 M H z}, \mathbf{C D C l}_{\mathfrak{3}}\right) \delta(\mathbf{p p m}): 0.82(\mathrm{t}, \mathrm{J}=7.33 \mathrm{~Hz}, 3 \mathrm{H}), 0.83(\mathrm{t}, \mathrm{J}=6.87 \mathrm{~Hz}, 3 \mathrm{H}), 1.0-1.3$ (m, 10H), $1.52(\mathrm{~m}, 2 \mathrm{H}), 1.60(\mathrm{~m}, 2 \mathrm{H}), 2.44(\mathrm{~m}, 1 \mathrm{H}), 7.14(\mathrm{~d}, \mathrm{~J}=7.33 \mathrm{~Hz}, 2 \mathrm{H}), 7.17$ (t, J = $7.33 \mathrm{~Hz}, 1 \mathrm{H}), 7.27$ (t, J = 7.33 Hz, 2H).

${ }^{13} \mathbf{C}\left(\mathbf{1 0 0 M H z}, \mathbf{C D C l}_{3}\right) \delta(\mathbf{p p m}): 14.03,14.08,22.56,22.79,27.29,29.85,31.98,36.69$, 36.94, 46.03, 125.64, 127.65 (2C), 128.10 (2C), 146.44 .

Micro-analysis : calcd (\%) : $\mathrm{C}=88.00, \mathrm{H}=12.00$ found $(\%): \mathrm{C}=88.28, \mathrm{H}=11.72$

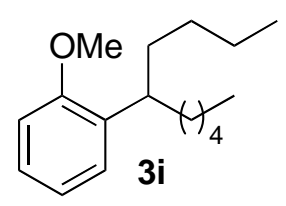

${ }^{1} \mathbf{H}\left(\mathbf{4 0 0 M H z}, \mathbf{C D C l}_{3}\right) \delta(\mathbf{p p m}): 0.75(\mathrm{t}, \mathrm{J}=7.33 \mathrm{~Hz}, 3 \mathrm{H}), 0.76(\mathrm{t}, \mathrm{J}=6.87 \mathrm{~Hz}, 3 \mathrm{H}), 1.0-1.25$ $(\mathrm{m}, 10 \mathrm{H}), 1.49(\mathrm{~m}, 4 \mathrm{H}), 3.00(\mathrm{~m}, 1 \mathrm{H}), 3.72(\mathrm{~s}, 3 \mathrm{H}), 6.77$ (d, J = $7.79 \mathrm{~Hz}, 1 \mathrm{H}), 6.83$ (t, J = $7.33 \mathrm{~Hz}, 1 \mathrm{H}), 7.04$ (d, J = 7.79 Hz, 1H), 7.06 (t, J = 7.79 Hz, 1H).

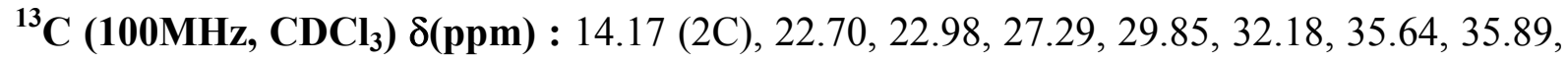
$37.37,55.56,110.63,120.59,126.33,127.52,134.86,157.77$. 
Micro-analysis : calcd (\%) : $\mathrm{C}=82.20, \mathrm{H}=11.36, \mathrm{O}=6.44$

found $(\%): \mathrm{C}=82.31, \mathrm{H}=11.22, \mathrm{O}=6.47$

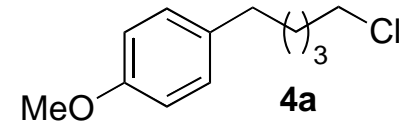

${ }^{1} \mathbf{H}\left(\mathbf{4 0 0 M H z}, \mathbf{C D C l}_{3}\right.$ ) $\delta(\mathbf{p p m}): 1.48$ (quint. , $\left.\mathrm{J}=7.33 \mathrm{~Hz}, 2 \mathrm{H}\right), 1.62$ (quint. , $\mathrm{J}=7.33 \mathrm{~Hz}$, 2H), 1.80 (quint. , $\mathrm{J}=7.33 \mathrm{~Hz}, 2 \mathrm{H}), 2.57$ (t, $\mathrm{J}=7.33 \mathrm{~Hz}, 2 \mathrm{H}), 3.53$ (t, $\mathrm{J}=7.33 \mathrm{~Hz}, 2 \mathrm{H}), 3.79$ $(\mathrm{s}, 3 \mathrm{H}), 6.83(\mathrm{~d}, \mathrm{~J}=7.79 \mathrm{~Hz}, 2 \mathrm{H}), 7.10(\mathrm{~d}, \mathrm{~J}=7.79 \mathrm{~Hz}, 2 \mathrm{H})$.

${ }^{13} \mathbf{C}\left(\mathbf{1 0 0 M H z}, \mathbf{C D C l}_{3}\right) \delta(\mathbf{p p m}) \quad: 26.44,30.98,32.47,34.77,45.04,55.20,113.63$, 129.20(2C), $134.36(2 \mathrm{C}), 157.61$.

Micro-analysis : calcd (\%) : $\mathrm{C}=67.76, \mathrm{H}=8.06, \mathrm{O}=7.52, \mathrm{Cl}=16.67$

found $(\%): \mathrm{C}=67.75, \mathrm{H}=8.18, \mathrm{O}=7.41, \mathrm{Cl}=16.66$

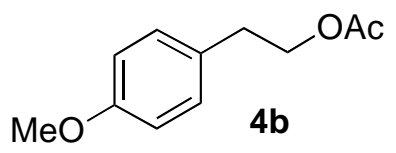

${ }^{1} \mathbf{H}\left(\mathbf{4 0 0 M H z}, \mathbf{C D C l}_{3}\right) \delta(\mathbf{p p m}): 1.98(\mathrm{~s}, 3 \mathrm{H}), 2.90(\mathrm{t}, \mathrm{J}=7.33 \mathrm{~Hz}, 2 \mathrm{H}), 3.79(\mathrm{~s}, 3 \mathrm{H}), 4.26(\mathrm{t}, \mathrm{J}$ $=7.33 \mathrm{~Hz}, 2 \mathrm{H}), 7.20(\mathrm{~d}, \mathrm{~J}=7.79 \mathrm{~Hz}, 2 \mathrm{H}), 7.27$ (d, J = 7.79 Hz, 2H).

${ }^{13} \mathbf{C}\left(\mathbf{1 0 0 M H z}, \mathbf{C D C l}_{3}\right) \delta(\mathbf{p p m}): 20.60,34.78,55.17,64.65,126.29,128.23$ (2C), 128.63 (2C), 137.54, 170.68 .

Micro-analysis : calcd (\%) : $\mathrm{C}=68.02, \mathrm{H}=7.27, \mathrm{O}=24.71$

found $(\%): \mathrm{C}=68.09, \mathrm{H}=7.04, \mathrm{O}=24.87$

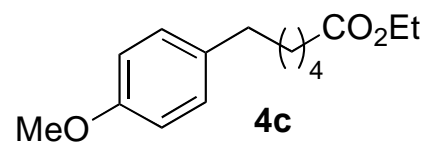

${ }^{1} \mathbf{H}\left(\mathbf{4 0 0 M H z}, \mathbf{C D C l}_{3}\right) \delta(\mathbf{p p m}): 1.24(\mathrm{t}, \mathrm{J}=7.33 \mathrm{~Hz}, 3 \mathrm{H}), 1.36(\mathrm{q}, \mathrm{J}=7.79 \mathrm{~Hz}, 2 \mathrm{H}), 1.62(\mathrm{~m}$, 4H), $2.30(\mathrm{t}, \mathrm{J}=7.33 \mathrm{~Hz}, 2 \mathrm{H}), 2.54(\mathrm{t}, \mathrm{J}=7.79 \mathrm{~Hz}, 2 \mathrm{H}), 3.79(\mathrm{~s}, 3 \mathrm{H}), 4.10$ (q, J = $7.33 \mathrm{~Hz}$, 2H), $6.82(\mathrm{~d}, \mathrm{~J}=8.20 \mathrm{~Hz}, 2 \mathrm{H}), 7.09$ (d, J = 8.70 Hz, 2H).

${ }^{13} \mathbf{C}\left(\mathbf{1 0 0 M H z}, \mathbf{C D C l}_{3}\right) \delta(\mathbf{p p m}): 14.18,24.75,28.59,31.30,34.20,34.70,55.13,60.12$, 113.54 (2C), 129.15 (2C), 134.50, 157.51, 173.76.

Micro-analysis : calcd (\%) : $\mathrm{C}=71.97, \mathrm{H}=8.86, \mathrm{O}=19.17$

found $(\%): \mathrm{C}=71.73, \mathrm{H}=9.07, \mathrm{O}=19.20$ 


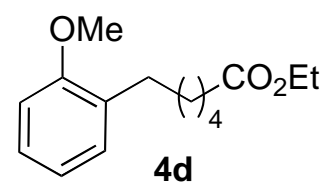

${ }^{1} \mathbf{H}\left(\mathbf{4 0 0 M H z}, \mathbf{C D C l}_{3}\right) \delta(\mathbf{p p m}): 1.17(\mathrm{t}, \mathrm{J}=7.33 \mathrm{~Hz}, 3 \mathrm{H}), 1.30$ (quint., $\left.\mathrm{J}=7.79 \mathrm{~Hz}, 2 \mathrm{H}\right), 1.52$ (quint., $\mathrm{J}=7.79 \mathrm{~Hz}, 2 \mathrm{H}), 1.59$ (quint., $\mathrm{J}=7.79 \mathrm{~Hz}, 2 \mathrm{H}), 2.22(\mathrm{t}, \mathrm{J}=7.79 \mathrm{~Hz}, 2 \mathrm{H}), 2.52(\mathrm{t}, \mathrm{J}=$ $7.79 \mathrm{~Hz}, 2 \mathrm{H}), 3.74$ (s, 3H), 4.05 (q, J = 7.33 Hz, 2H), 6.76 (d, J = 7.79 Hz, 1H), 6.80 (t, J = $7.33 \mathrm{~Hz}, 1 \mathrm{H}), 7.04$ (d, J = 7.33 Hz, 1H), 7.09 (t, J = 7.79 Hz, 1H).

${ }^{13} \mathbf{C}\left(\mathbf{1 0 0 M H z}, \mathbf{C D C l}_{3}\right) \delta(\mathbf{p p m}): 14.23,24.84,29.00,29.40,29.94,34.32,55.19,60.13$, $110.17,120.28,126.84,129.72,130.93,157.39,173.90$.

Micro-analysis : calcd (\%) : $\mathrm{C}=71.97, \mathrm{H}=8.86, \mathrm{O}=19.17$ found $(\%): \mathrm{C}=72.15, \mathrm{H}=8.93, \mathrm{O}=18.92$

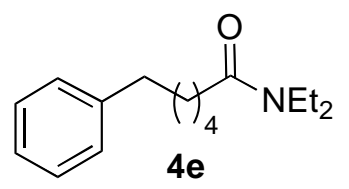

${ }^{1} \mathbf{H}\left(\mathbf{4 0 0 M H z}, \mathbf{C D C l}_{3}\right) \delta(\mathbf{p p m}): 1.12(\mathrm{t}, \mathrm{J}=7.33 \mathrm{~Hz}, 3 \mathrm{H}), 1.15(\mathrm{t}, \mathrm{J}=7.33 \mathrm{~Hz}, 3 \mathrm{H}), 1.38(\mathrm{~m}$, 2H), $1.65(\mathrm{~m}, 4 \mathrm{H}), 2.27(\mathrm{t}, \mathrm{J}=7.33 \mathrm{~Hz}, 2 \mathrm{H}), 2.61(\mathrm{t}, \mathrm{J}=7.79 \mathrm{~Hz}, 2 \mathrm{H}), 3.27(\mathrm{q}, \mathrm{J}=7.33 \mathrm{~Hz}$, 2H), 3.35 (q, J = 7.33 Hz, 2H), 7.17 (m, 3H), 7.26 (m, 2H).

${ }^{13} \mathbf{C}\left(\mathbf{1 0 0 M H z}, \mathbf{C D C l}_{3}\right) \delta(\mathbf{p p m}): 13.03,14.30,25.53,29.06,31.30,32.94,35.72,39.91$, $41.81,125.51,128.14(2 \mathrm{C}), 128.32(2 \mathrm{C}), 142.57,172.04$.

Micro-analysis : calcd (\%) : $\mathrm{C}=77.68, \mathrm{H}=10.19, \mathrm{O}=6.47, \mathrm{~N}=5.66$ found $(\%): \mathrm{C}=77.54, \mathrm{H}=10.57, \mathrm{O}=, \mathrm{N}=5.54$

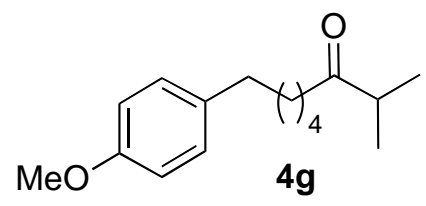

${ }^{1} \mathbf{H}\left(\mathbf{4 0 0 M H z}, \mathbf{C D C l}_{3}\right) \delta(\mathbf{p p m}): 1.07(\mathrm{~d}, \mathrm{~J}=6.87 \mathrm{~Hz}, 6 \mathrm{H}), 1.31$ (quint. , $\left.\mathrm{J}=7.33 \mathrm{~Hz}, 2 \mathrm{H}\right)$, 1.58 (quint. , $\mathrm{J}=7.79 \mathrm{~Hz}, 4 \mathrm{H}), 2.41(\mathrm{t}, \mathrm{J}=6.87 \mathrm{~Hz}, 2 \mathrm{H}), 2.54(\mathrm{~m}, 3 \mathrm{H}), 3.77$ (s, 3H), 6.81 (d, J $=8.70 \mathrm{~Hz}, 2 \mathrm{H}), 7.07(\mathrm{~d}, \mathrm{~J}=8.70 \mathrm{~Hz}, 2 \mathrm{H})$. 


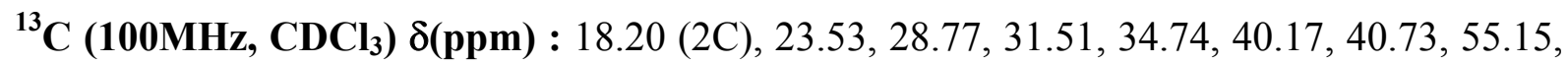
113.54 (2C), $129.16(2 \mathrm{C}), 134.59,157.51,215.00$.

Micro-analysis : $\operatorname{calcd}(\%): \mathrm{C}=77.38, \mathrm{H}=9.74, \mathrm{O}=12.88$ found $(\%): \mathrm{C}=77.59, \mathrm{H}=9.49, \mathrm{O}=12.92$

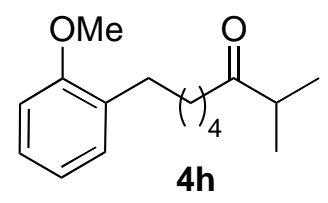

${ }^{1} \mathbf{H}\left(\mathbf{4 0 0 M H z}, \mathbf{C D C l}_{3}\right) \delta(\mathbf{p p m}): 0.99(\mathrm{~d}, \mathrm{~J}=6.87 \mathrm{~Hz}, 6 \mathrm{H}), 1.26$ (quint., $\left.\mathrm{J}=6.87 \mathrm{~Hz}, 2 \mathrm{H}\right), 1.53$ (m, 4H), 2.37 (t, J = 6.87 Hz, 2H), $2.53(\mathrm{t}, \mathrm{J}=6.87 \mathrm{~Hz}, 2 \mathrm{H}), 3.70(\mathrm{~m}, 1 \mathrm{H}), 3.72(\mathrm{~s}, 3 \mathrm{H}), 6.75$ (d, J = 7.79 Hz, 1H), 6.79 (t, J = 7.33 Hz, 1H), 7.03 (d, J = 7.33 Hz, 1H), 7.07 (t, J = 7.79 Hz, $1 \mathrm{H})$.

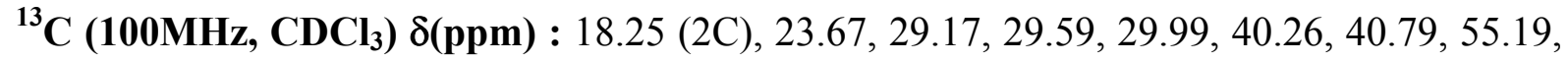
$110.17,120.27,126.83,129.73,130.98,157.42,215.09$.

Micro-analysis : calcd (\%) : $\mathrm{C}=77.38, \mathrm{H}=9.74, \mathrm{O}=12.88$ found $(\%): \mathrm{C}=77.71, \mathrm{H}=9.52, \mathrm{O}=12.77$

\section{4- References}

1. Watson, S. C.; Eastham, J. F. J. Organometal. Chem. 1967, 9, 165. 


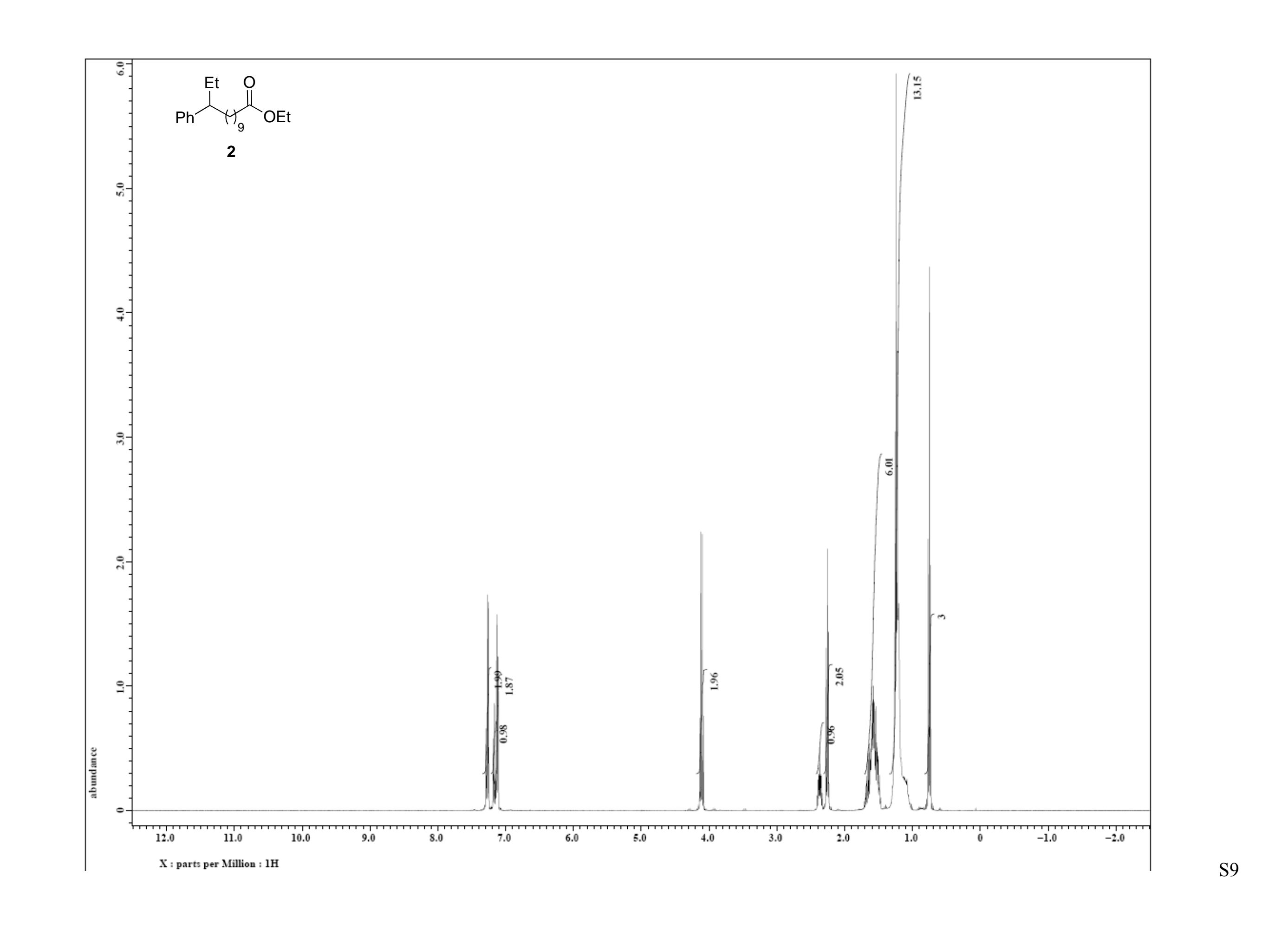




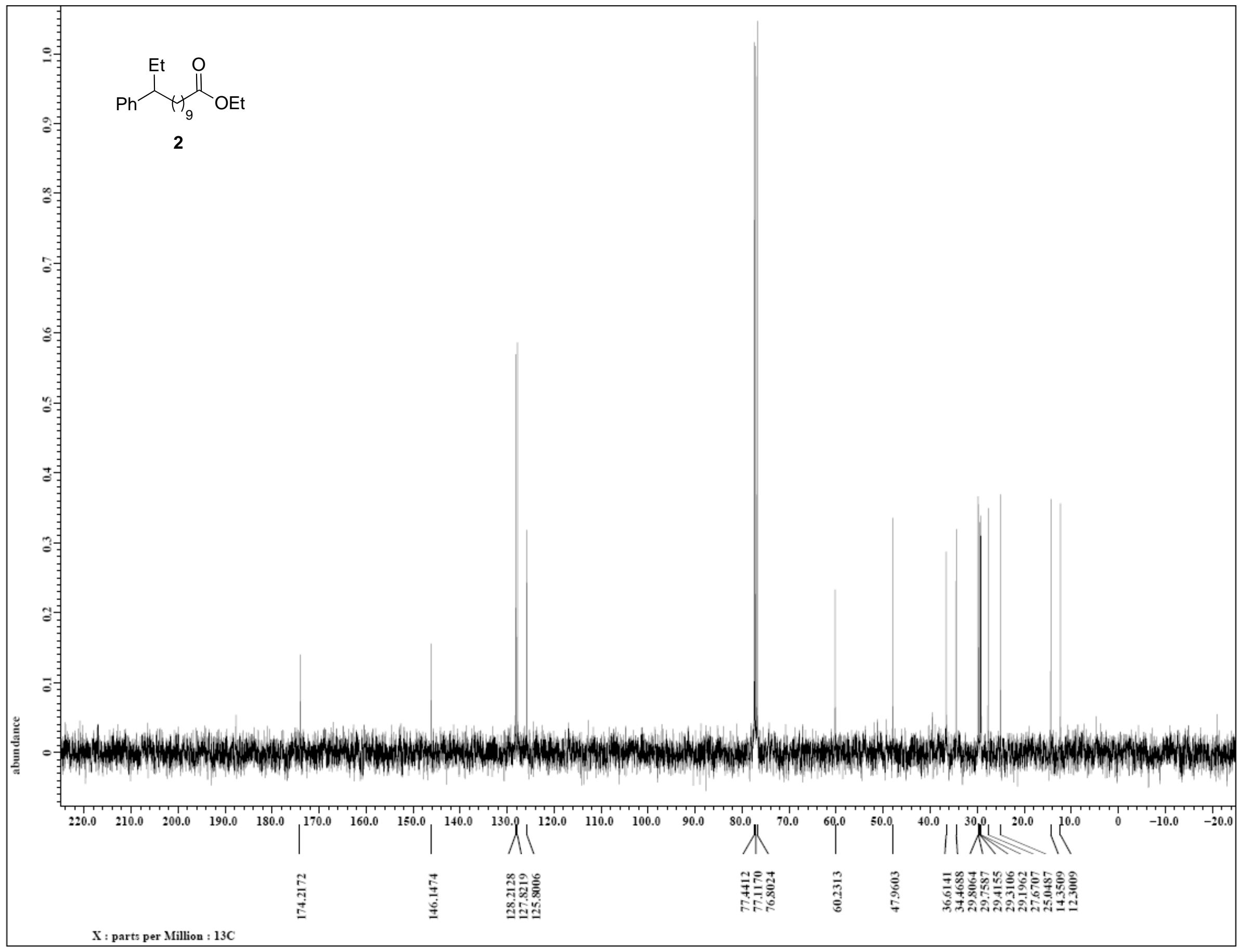




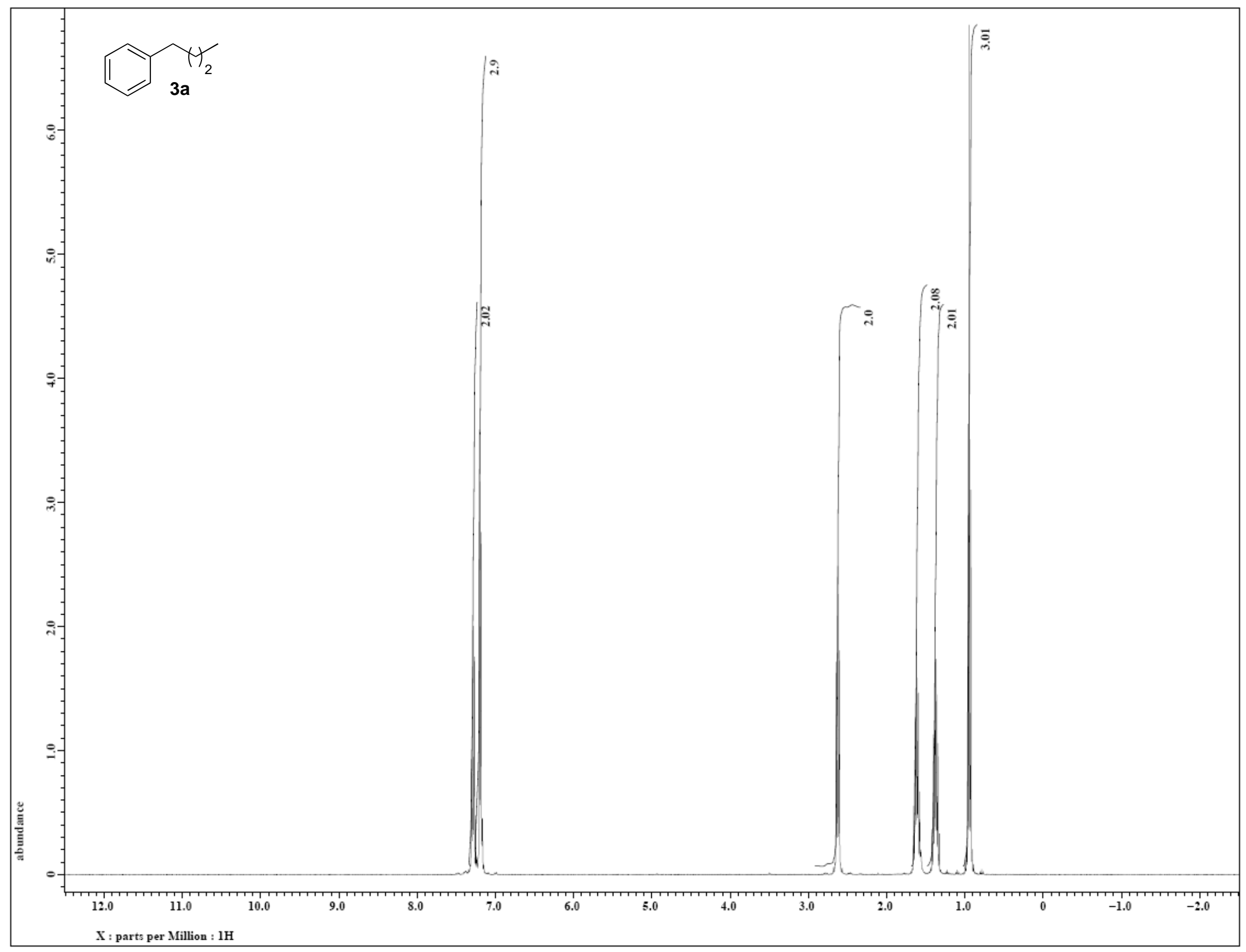




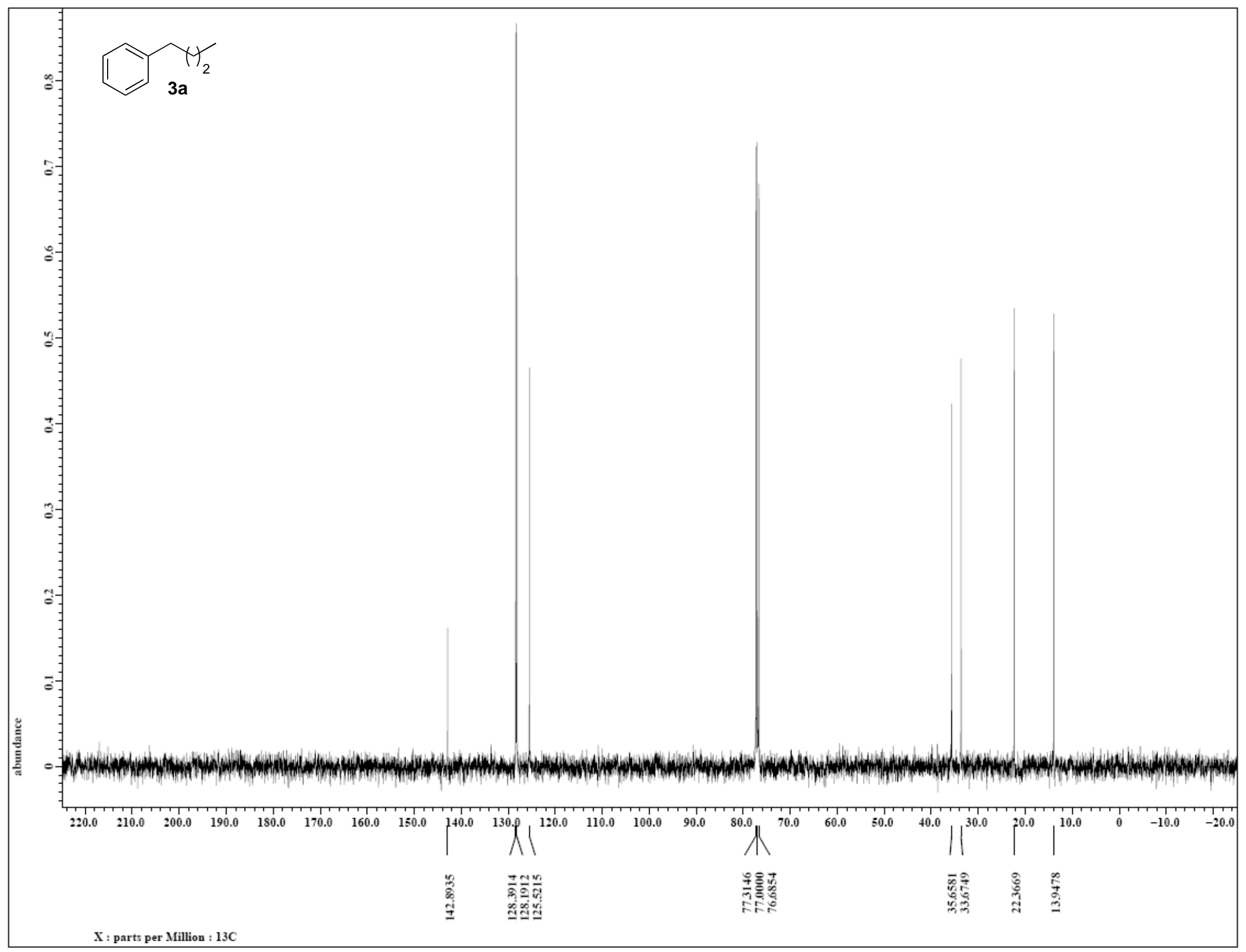




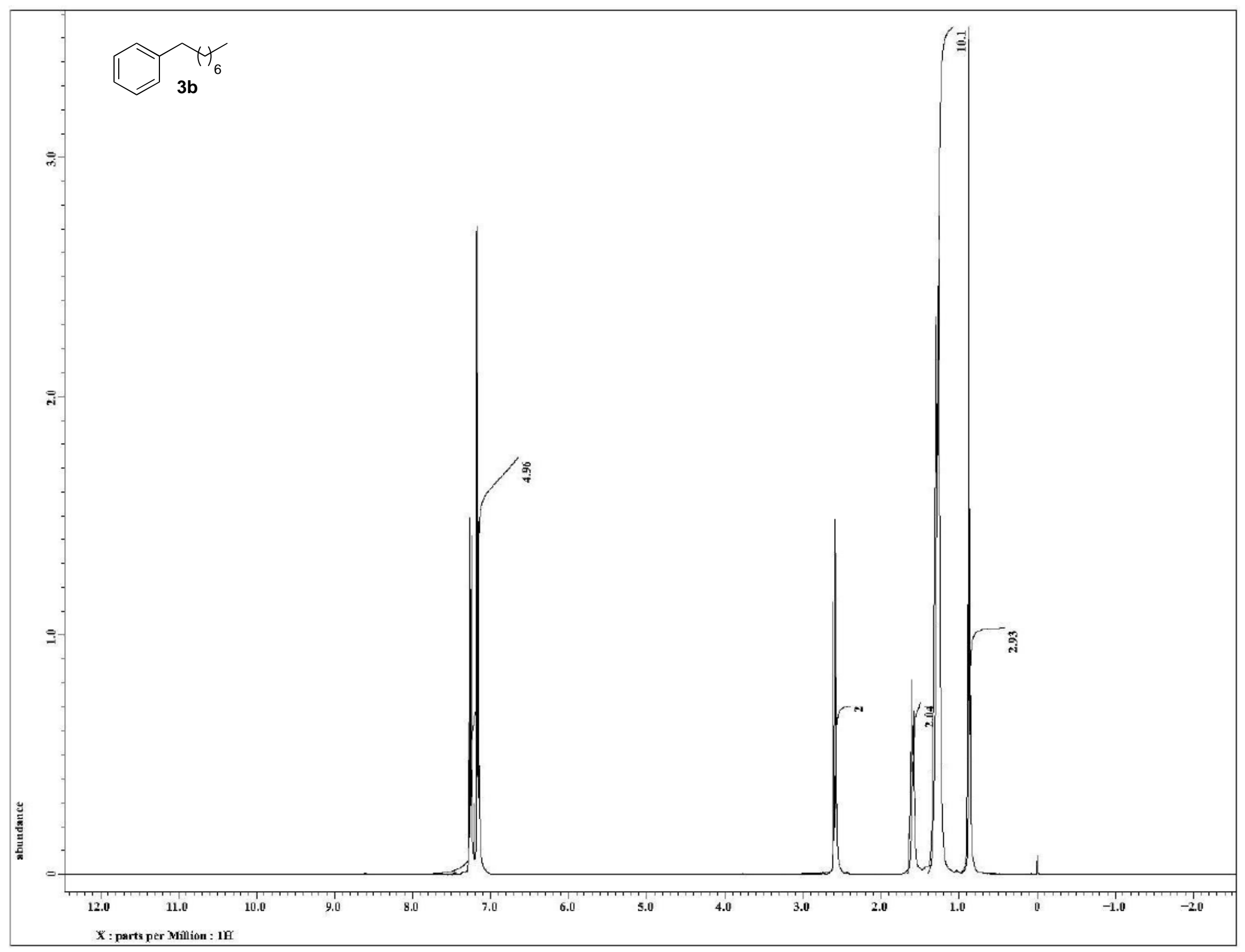




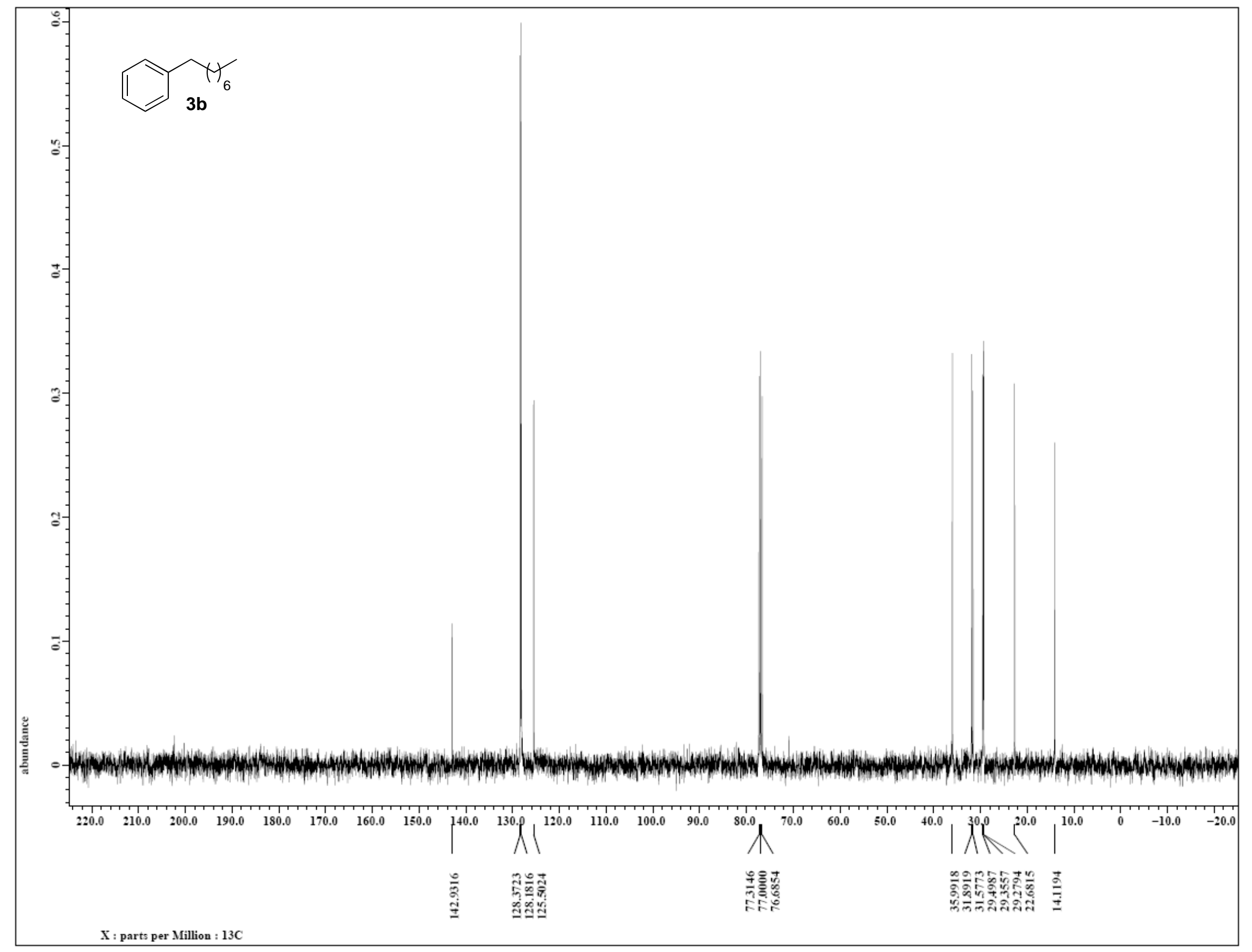




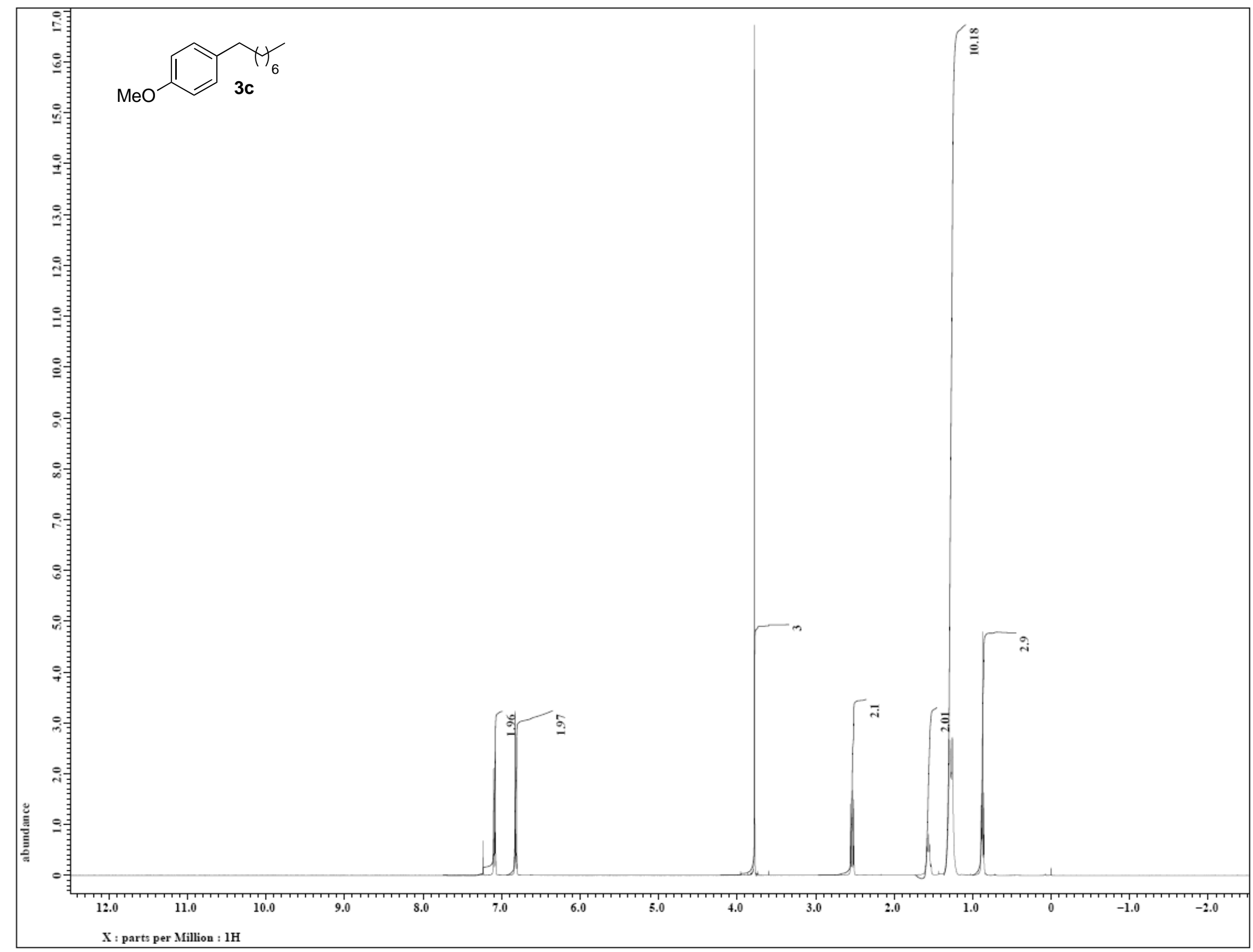




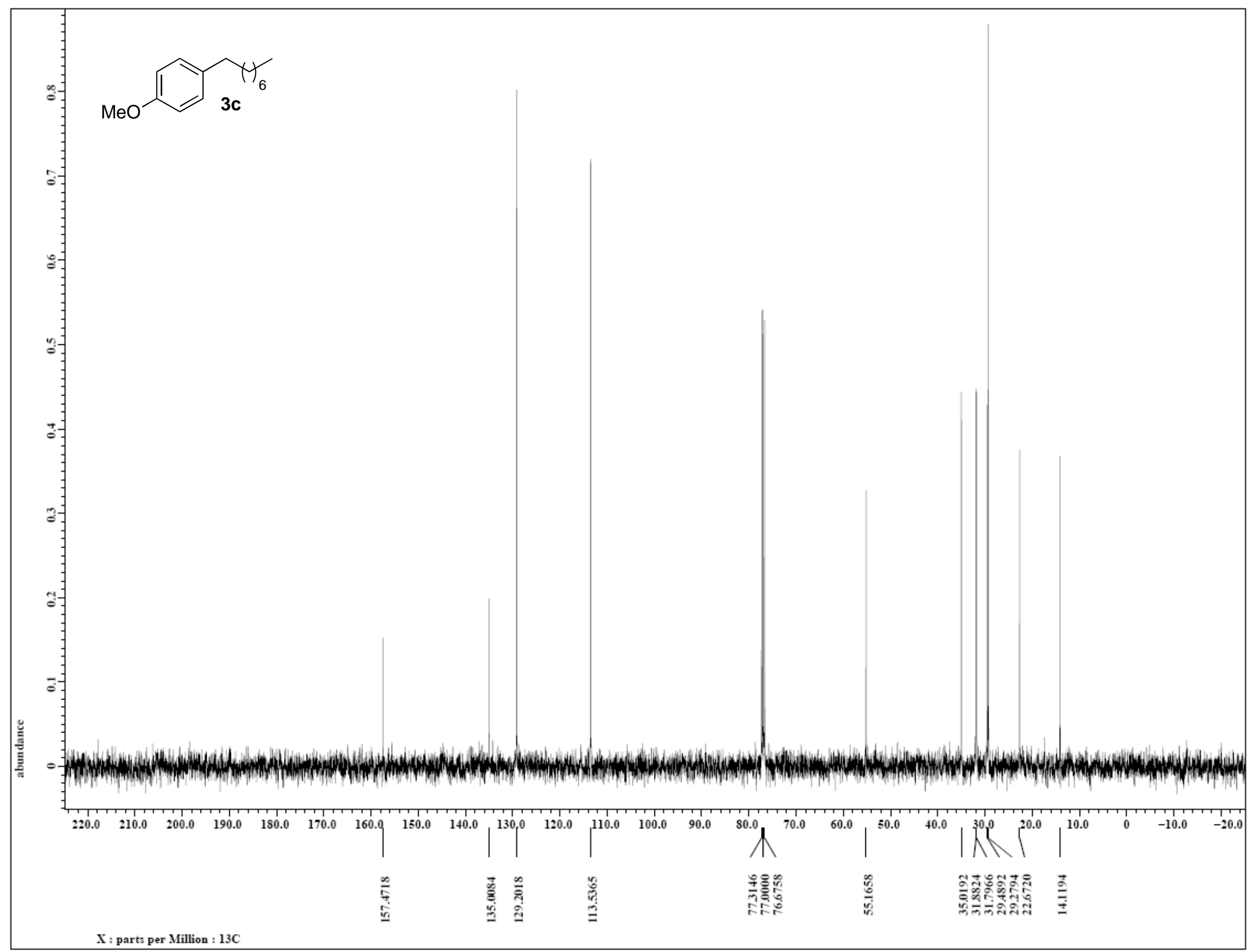




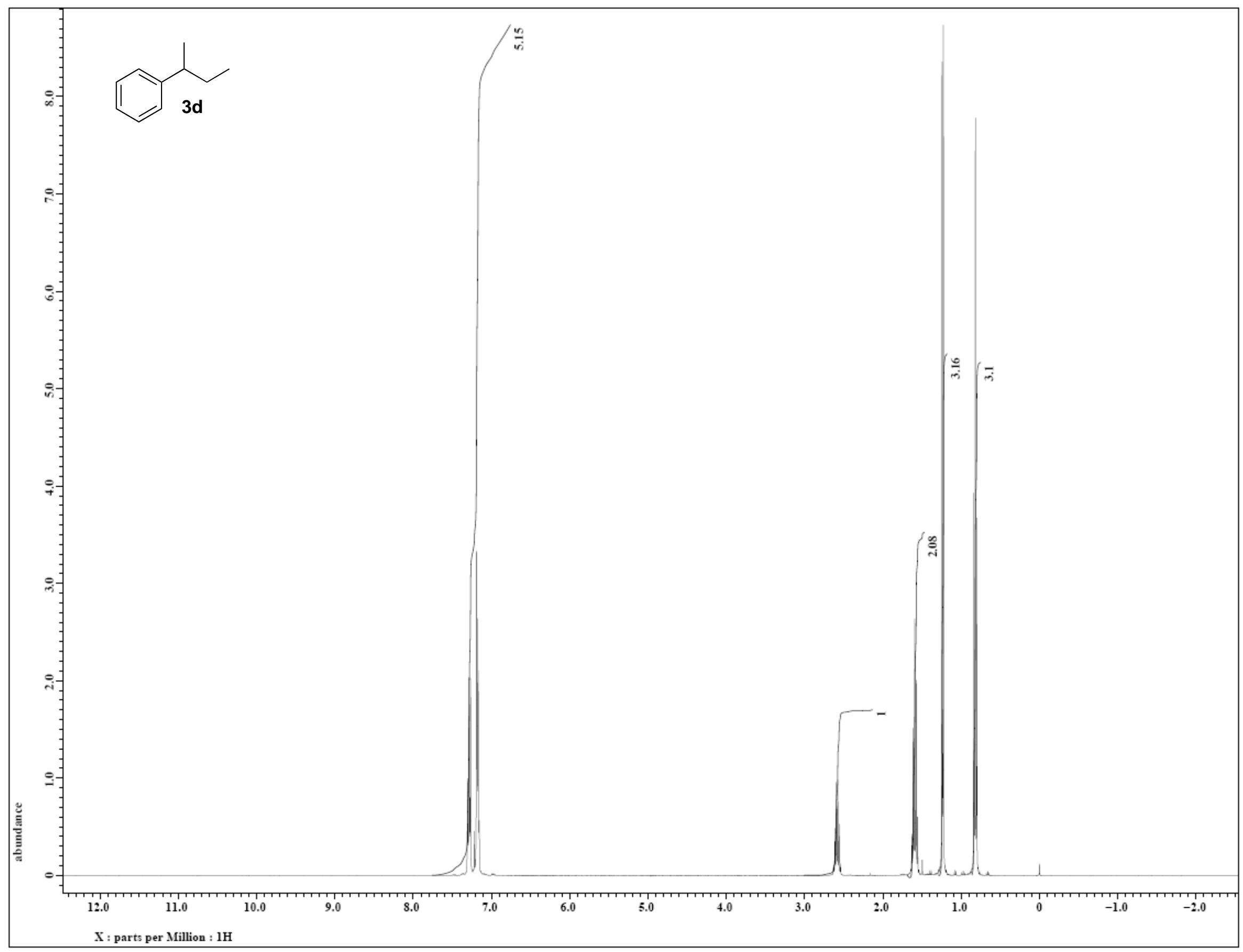




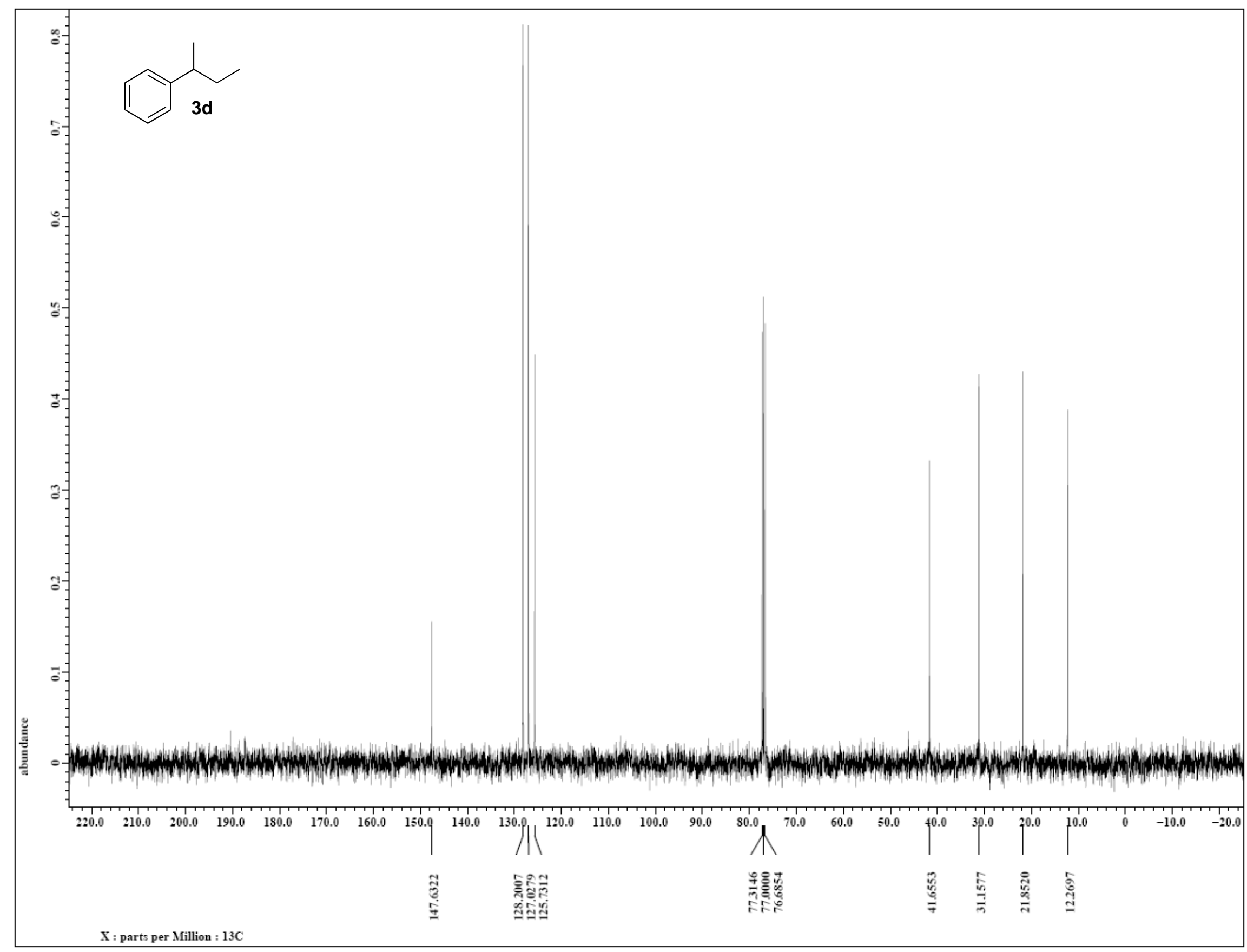




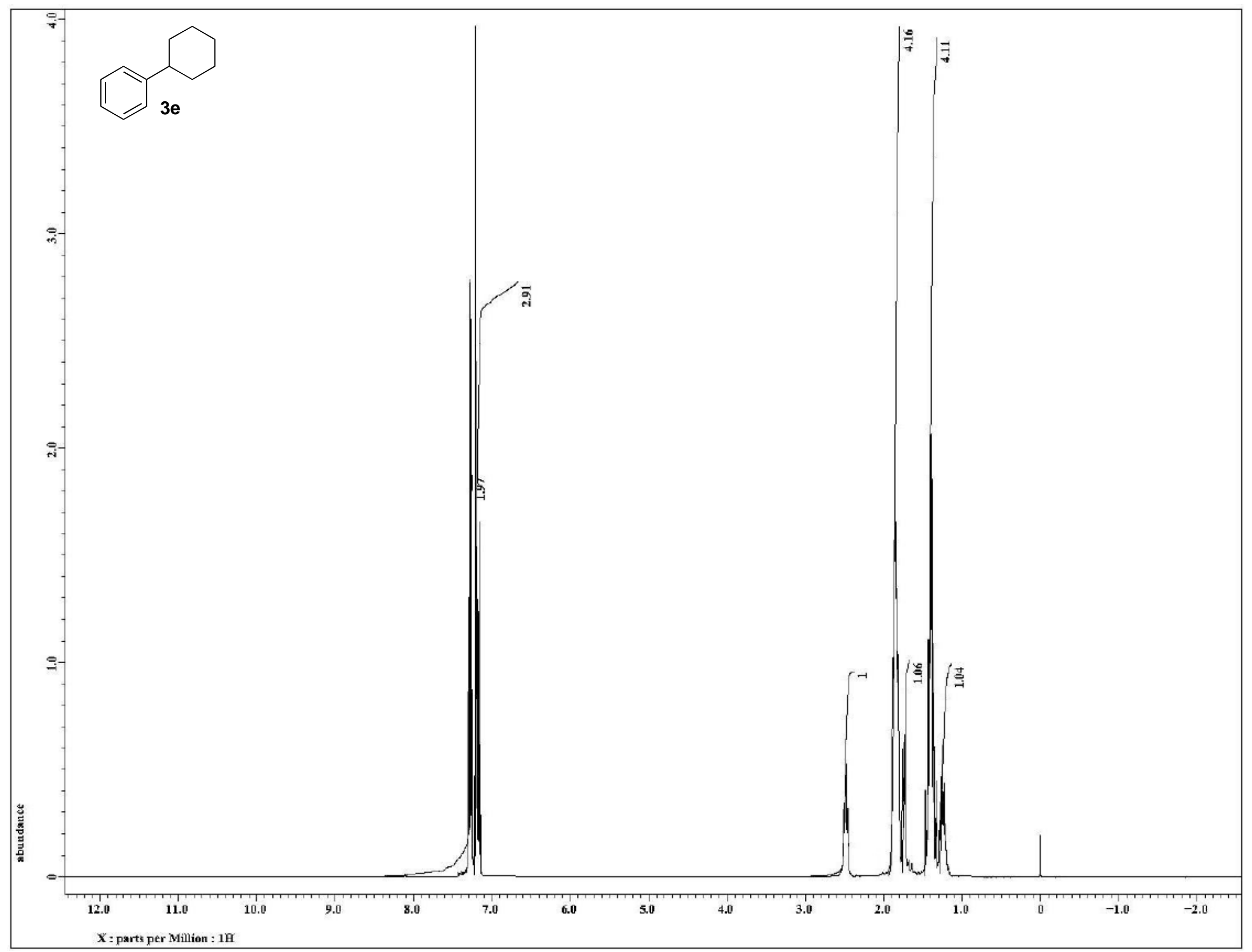




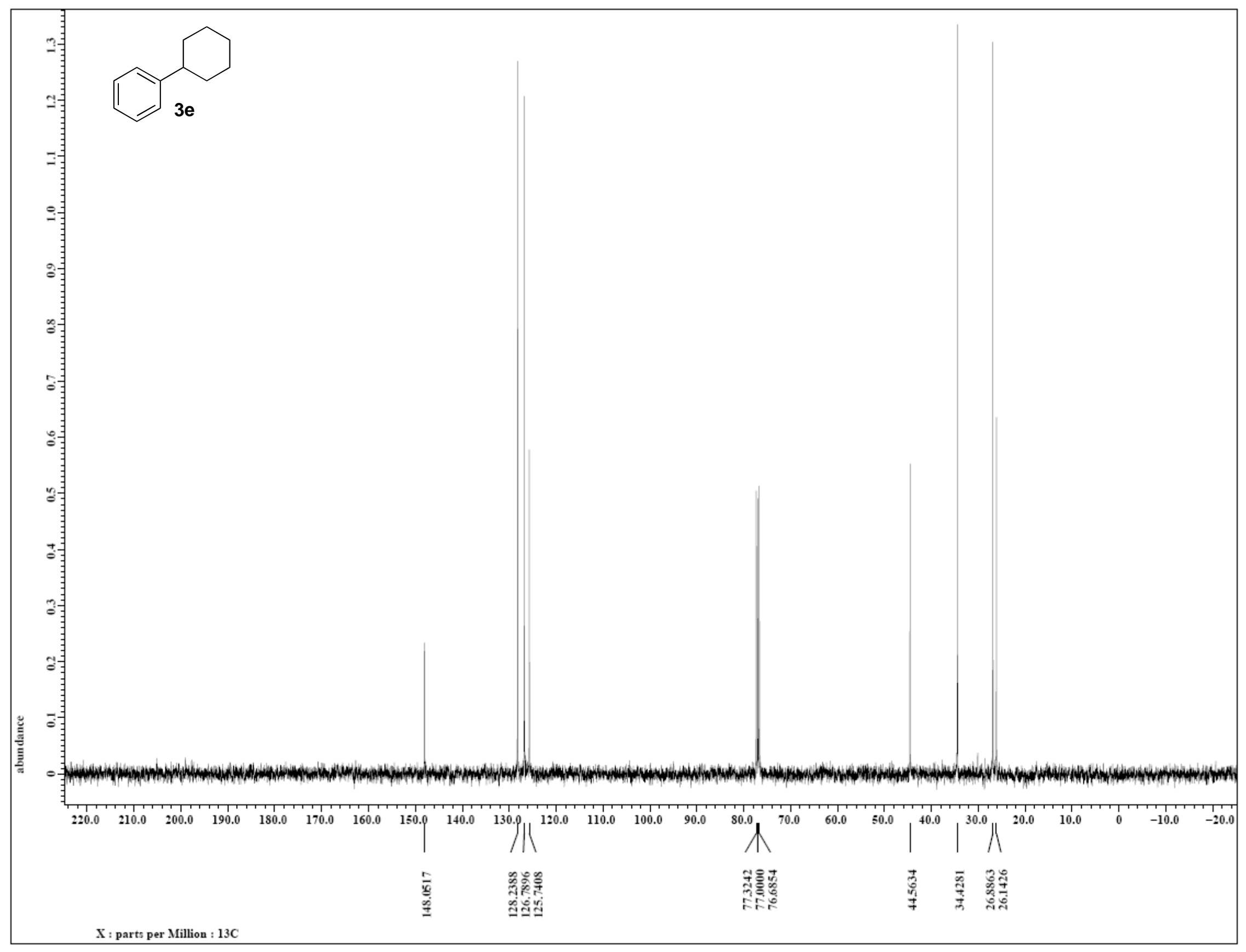




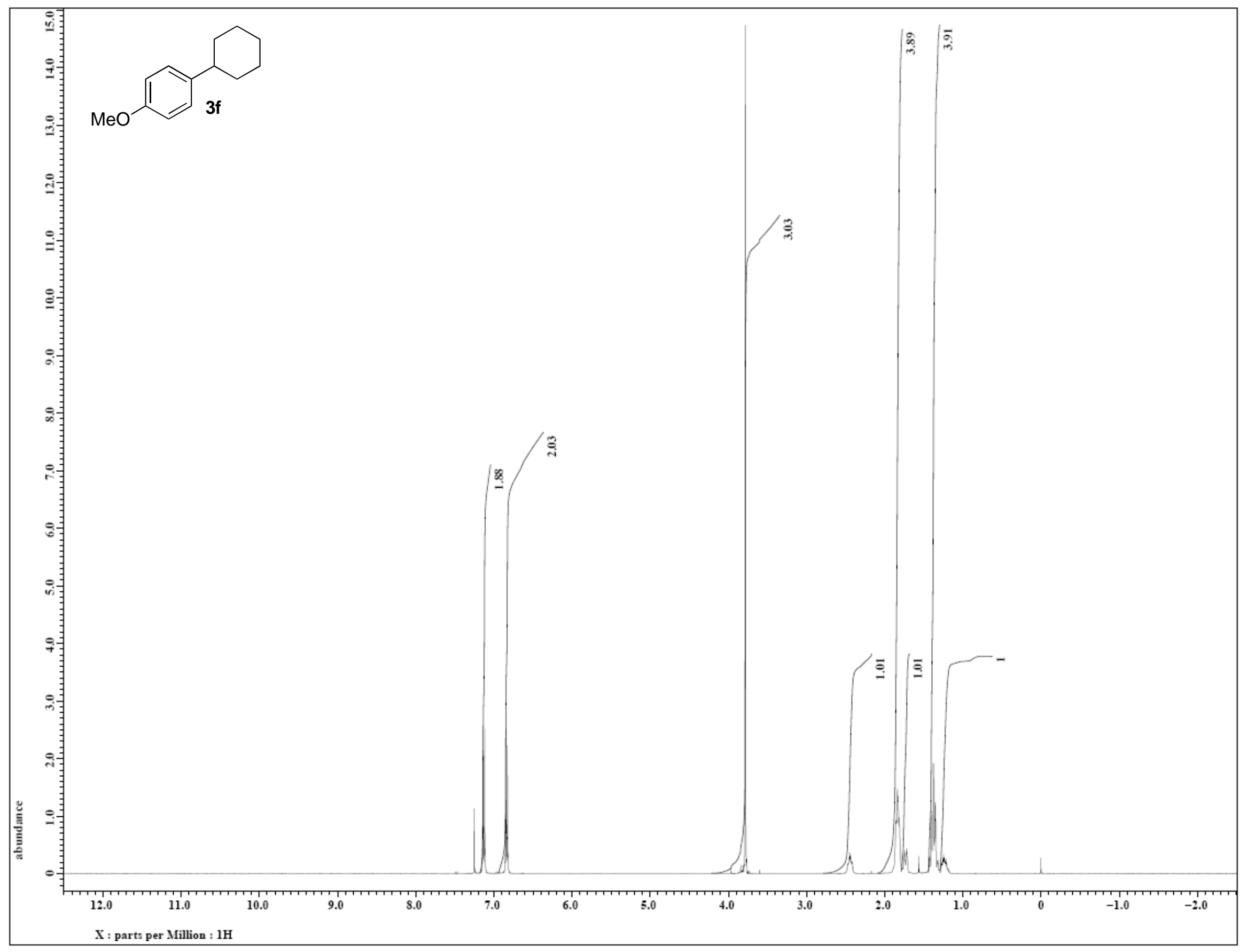




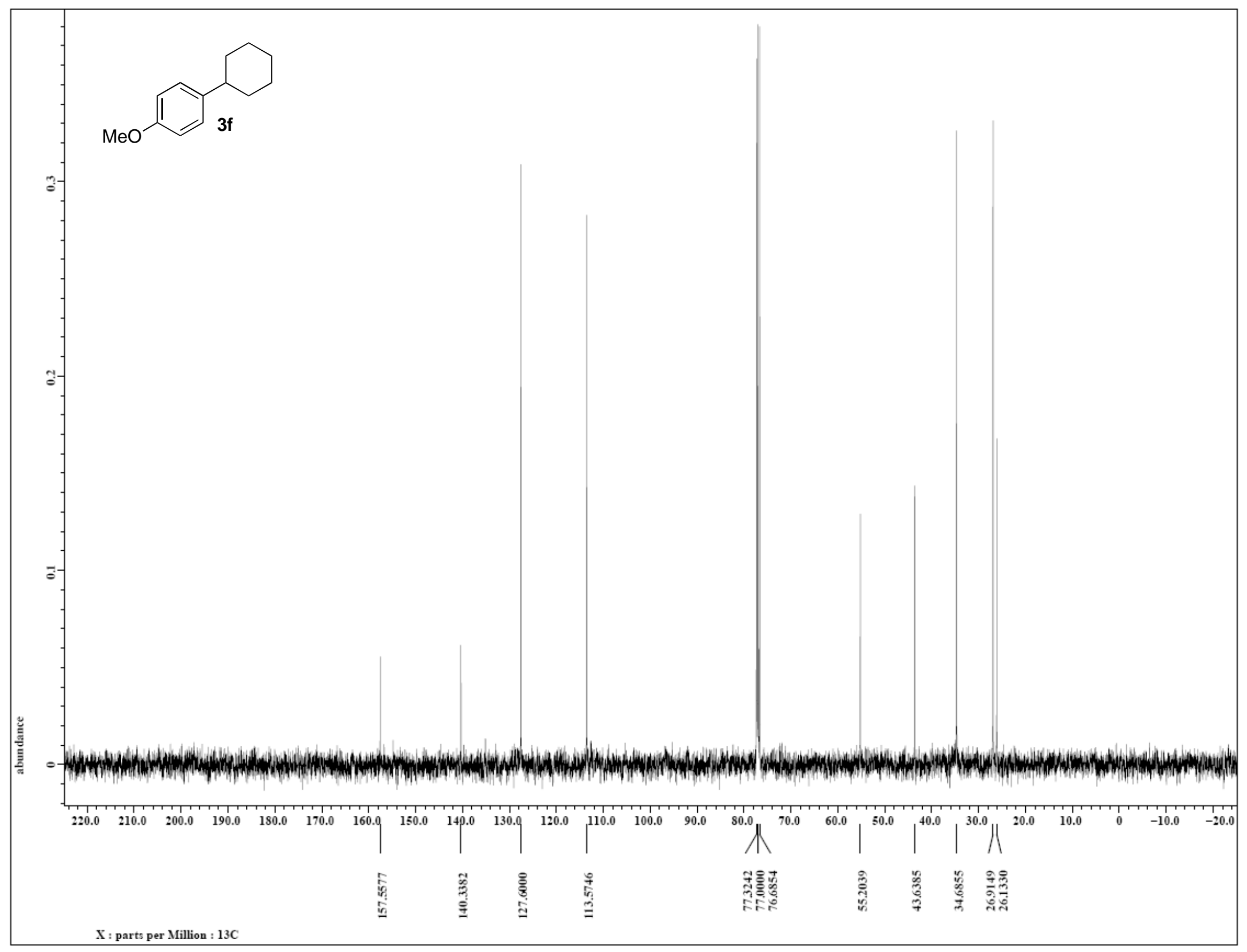




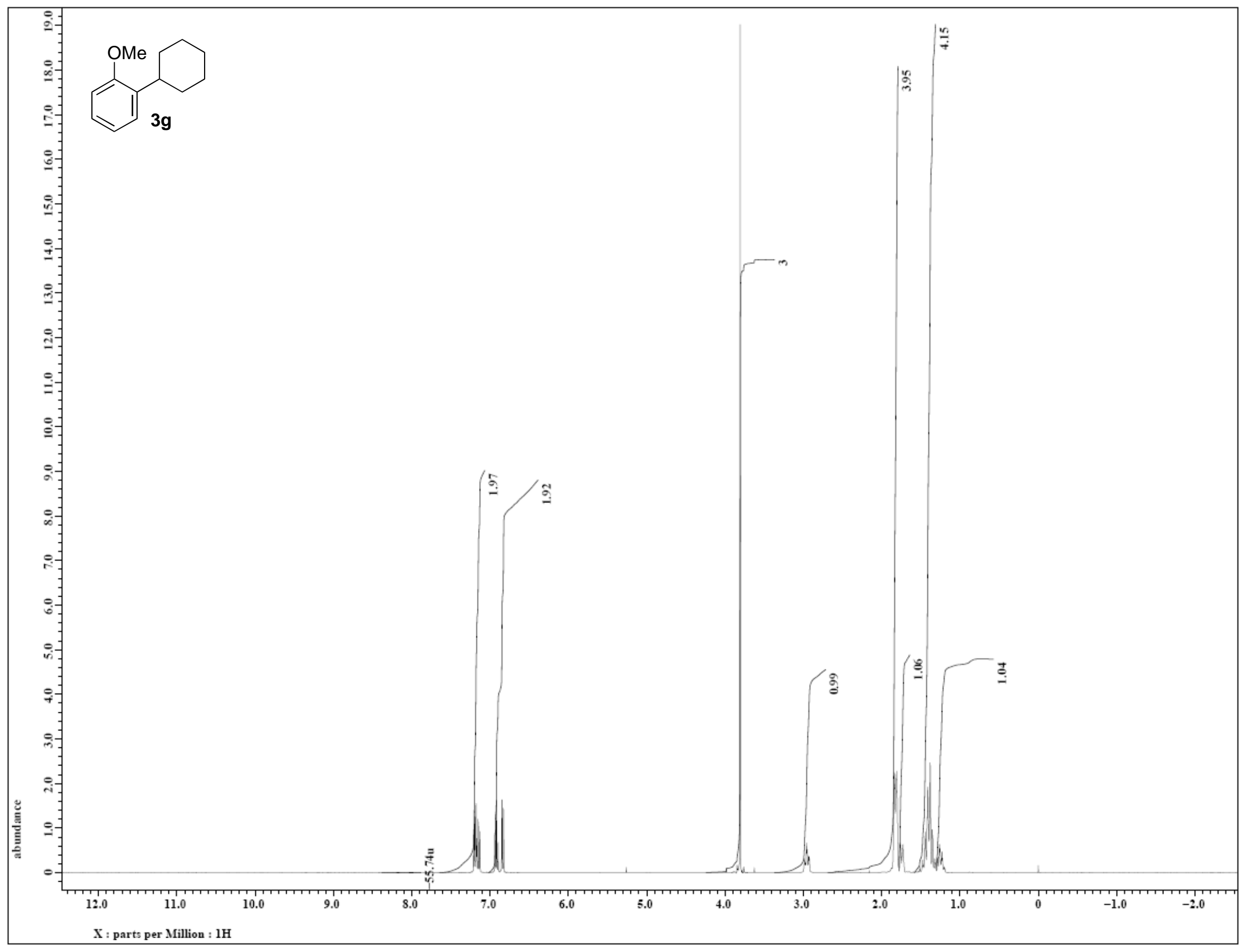




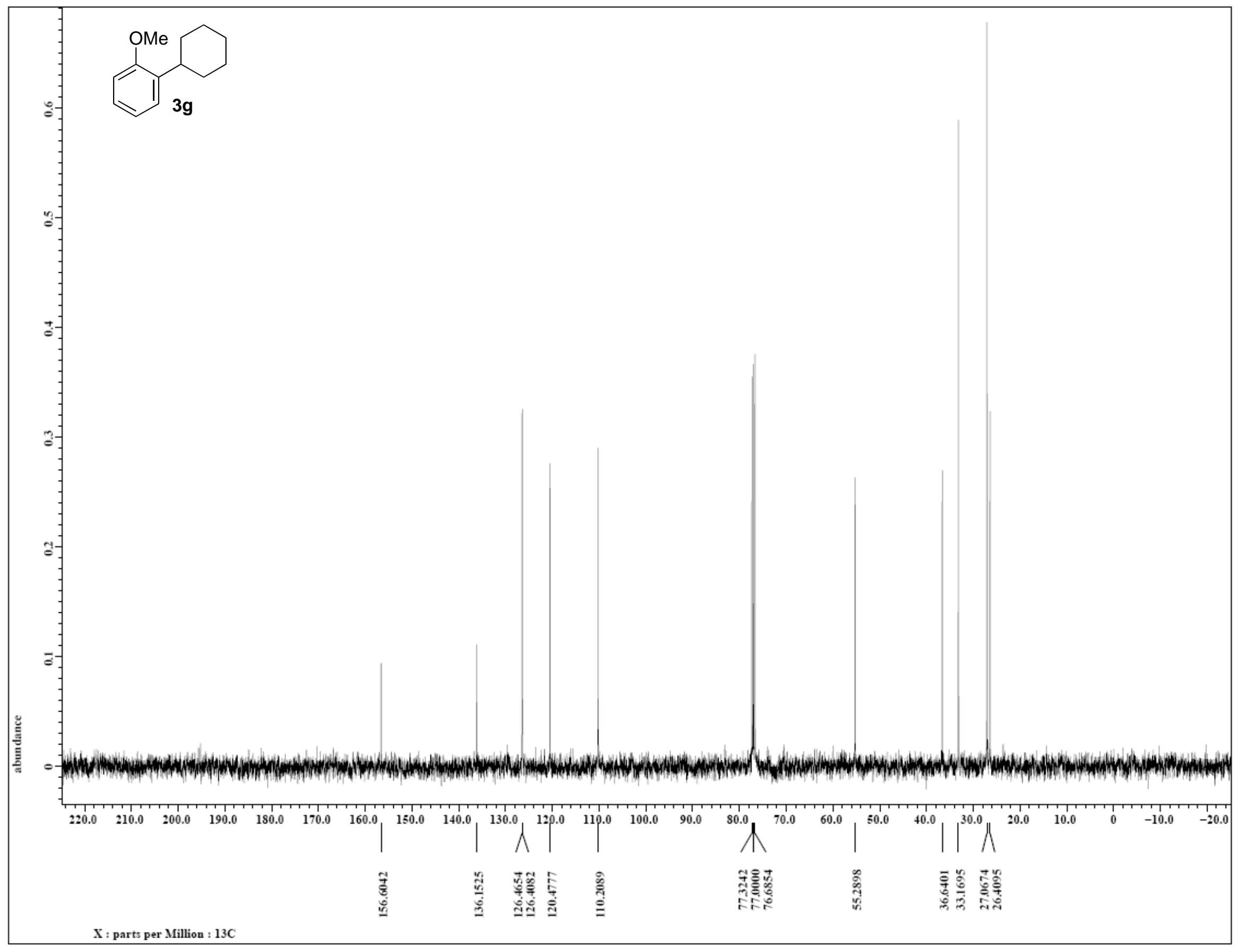




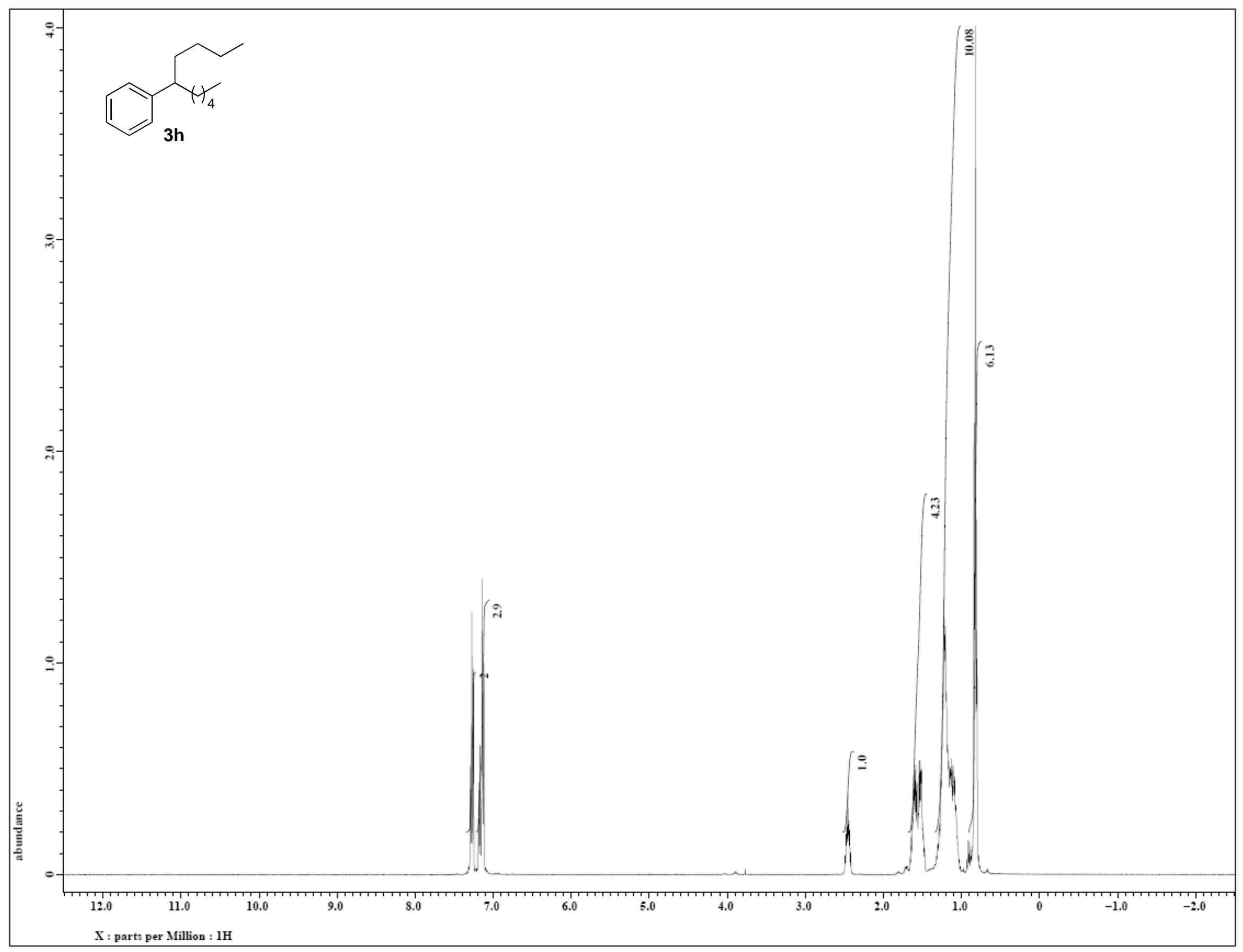




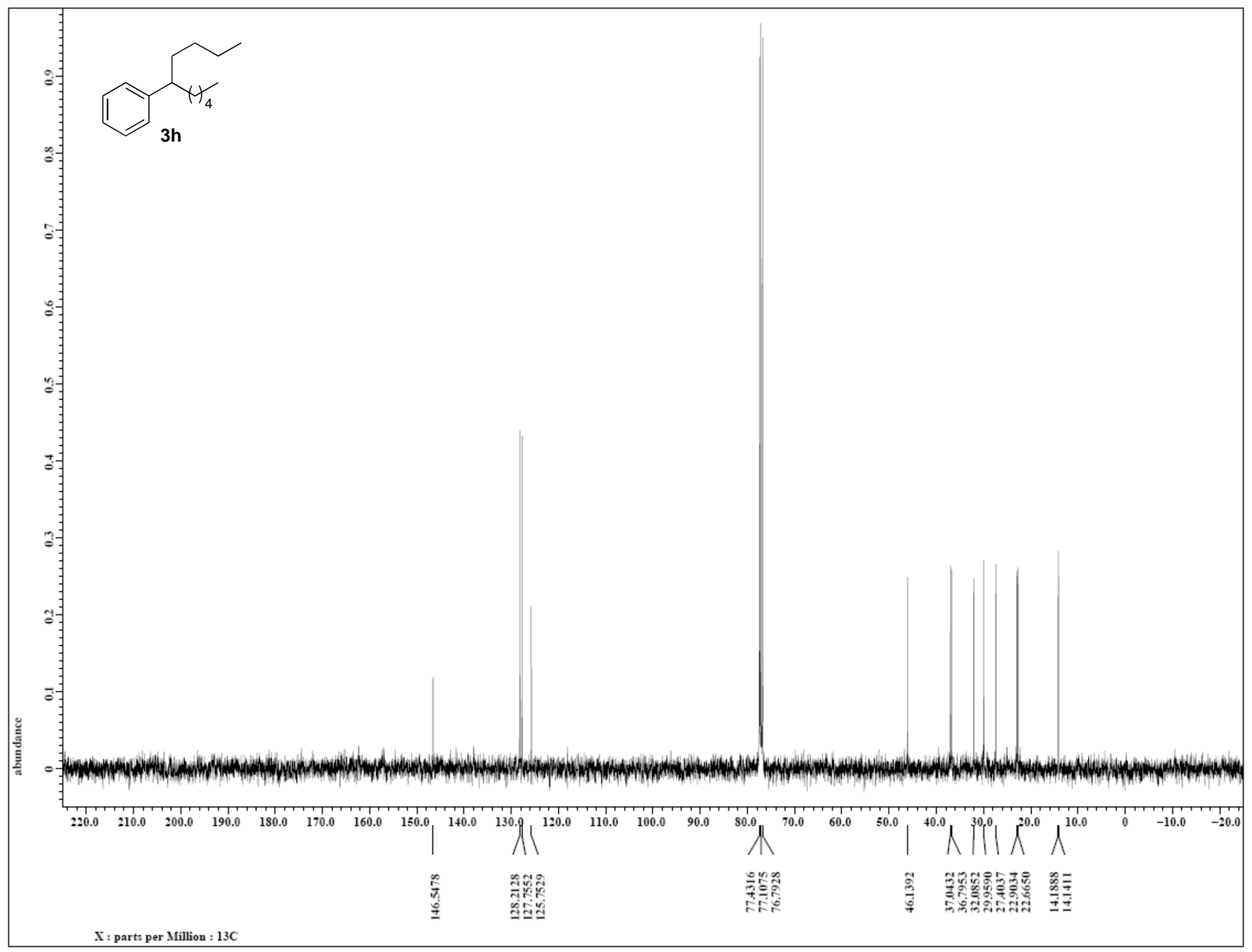




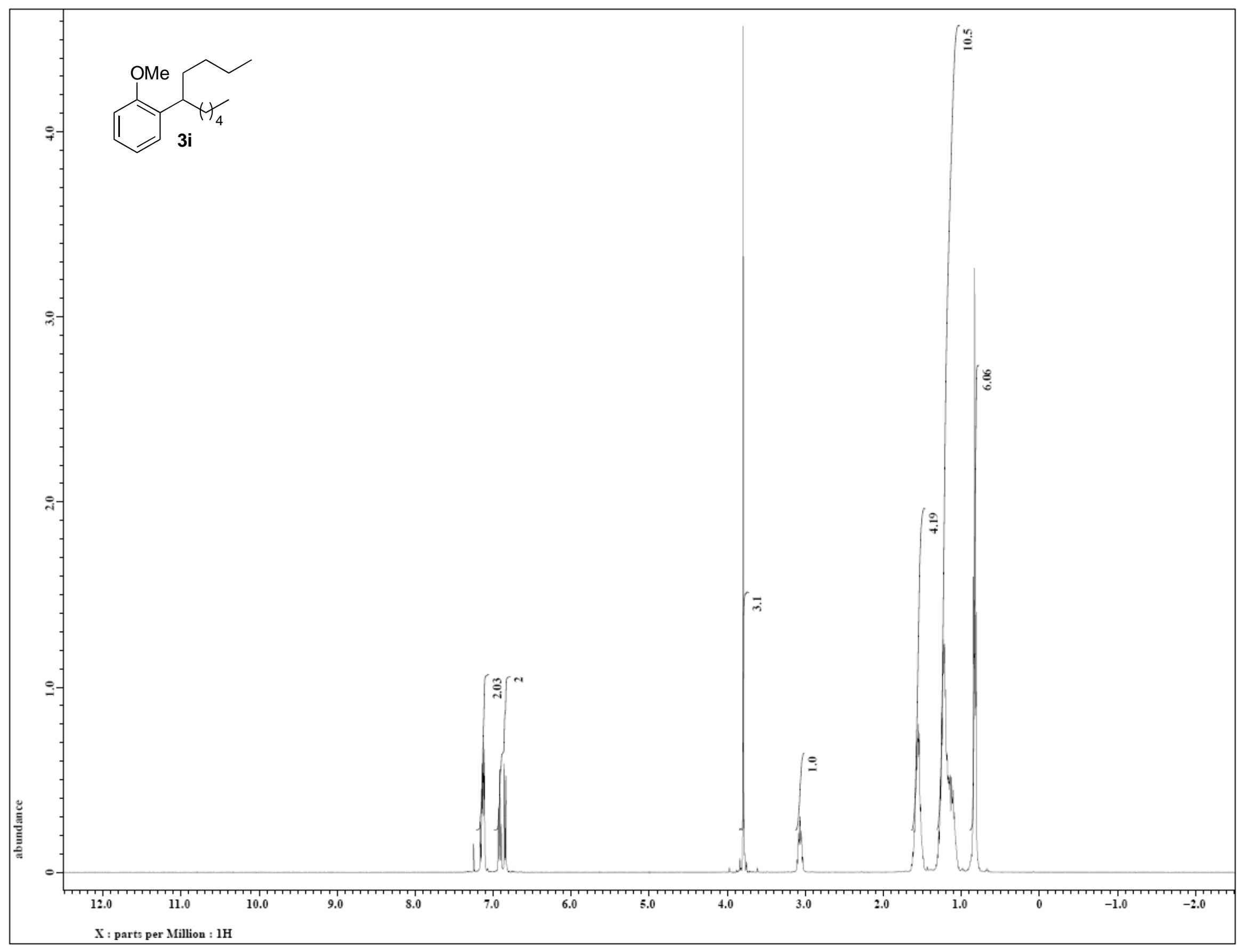




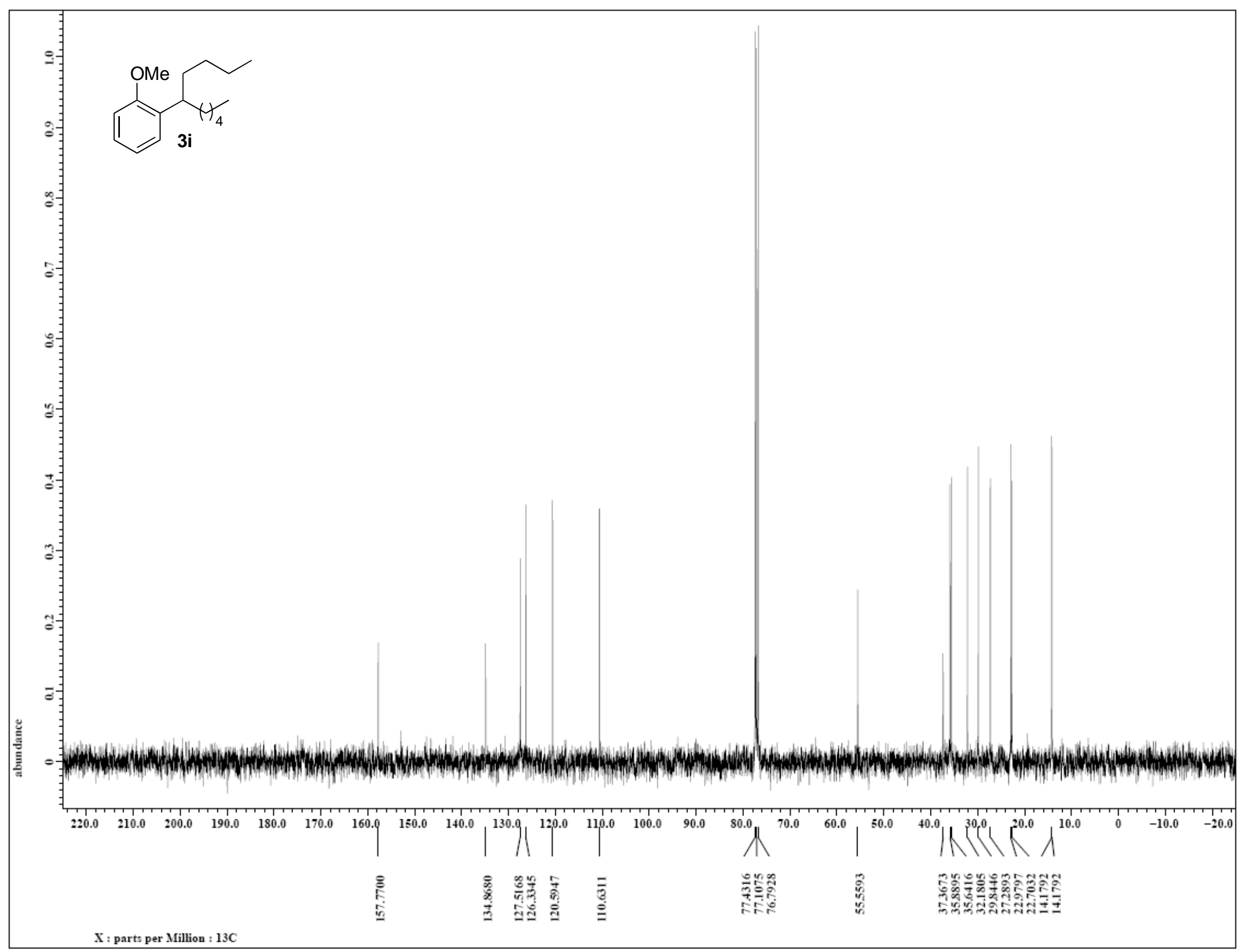




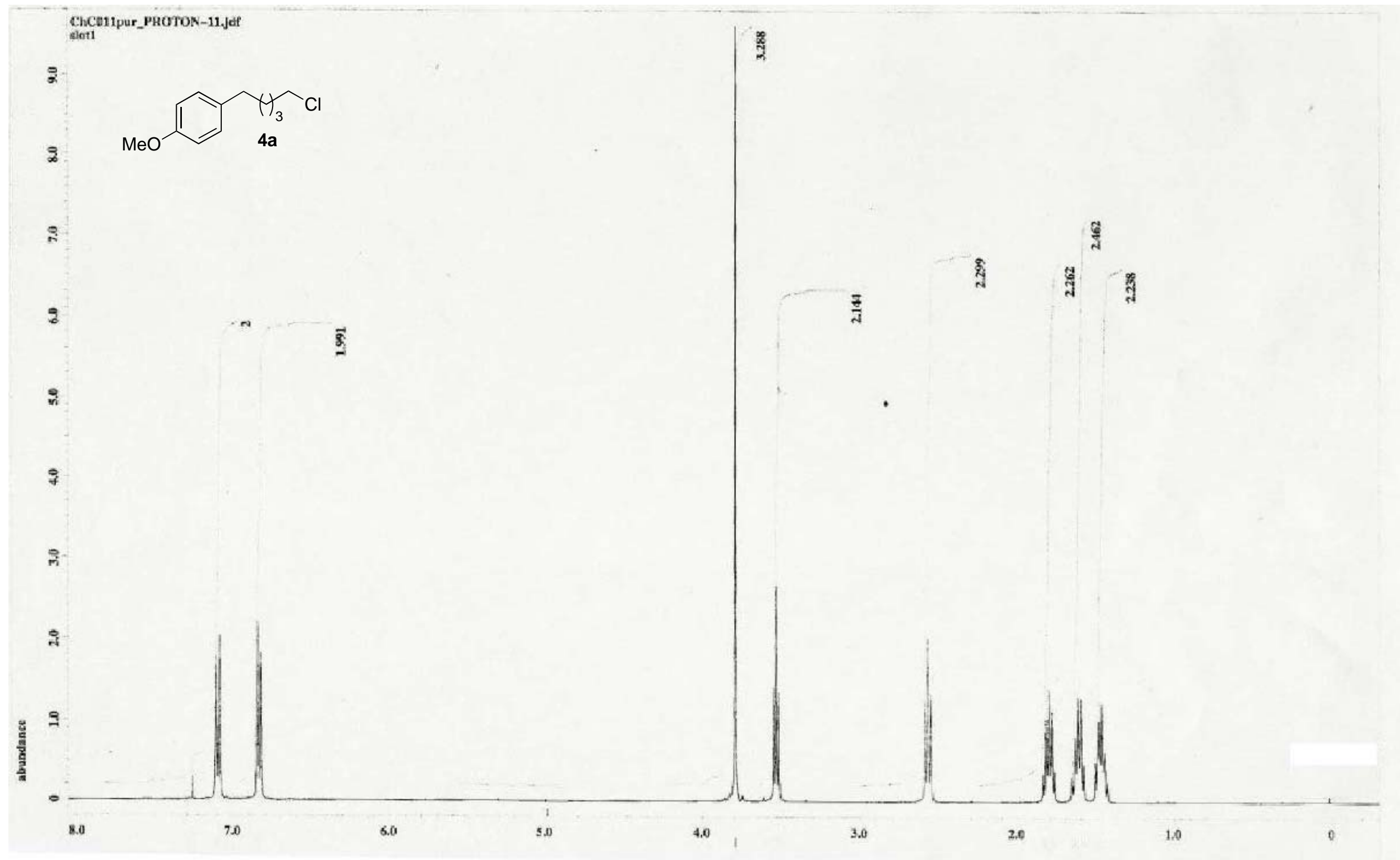




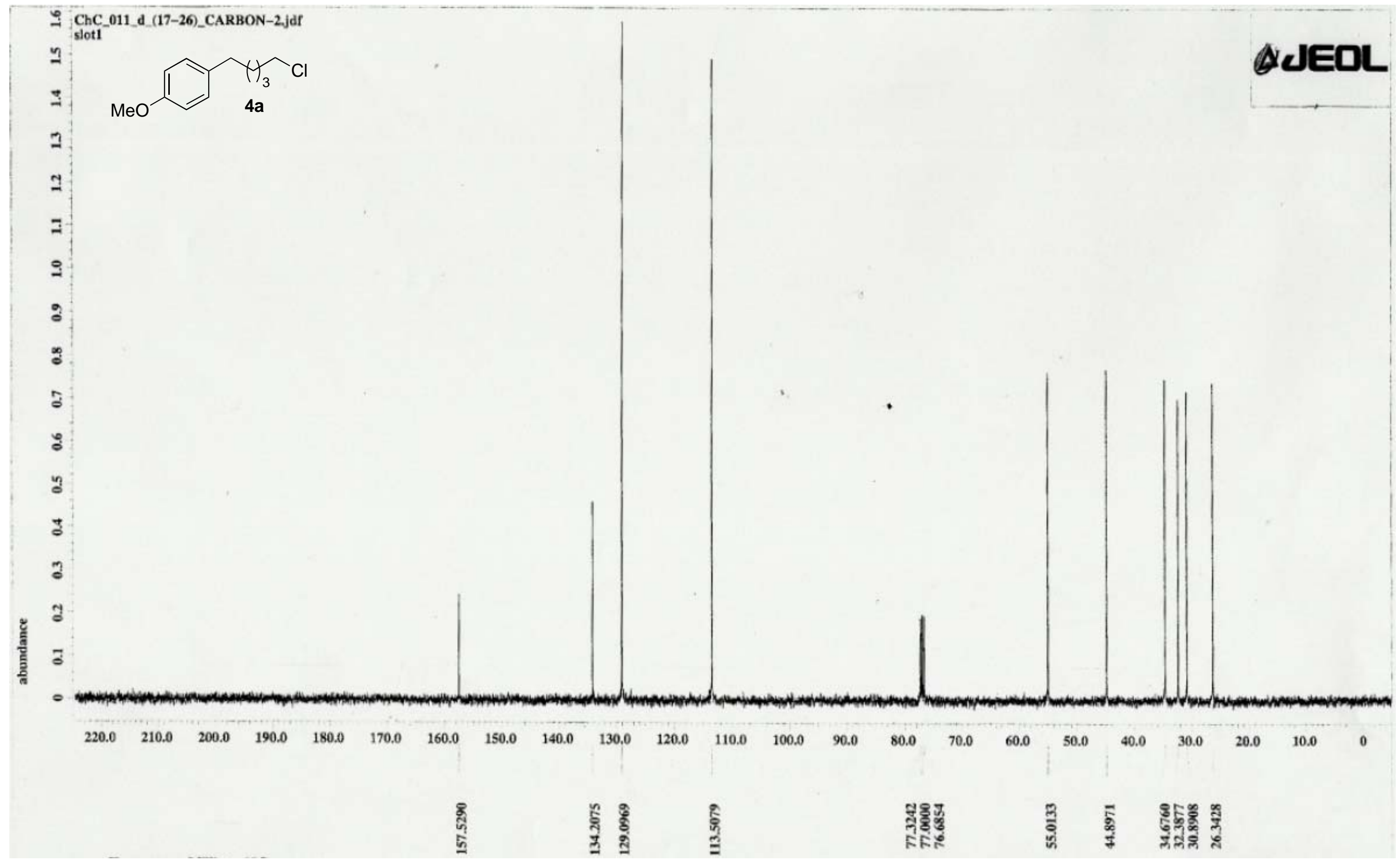




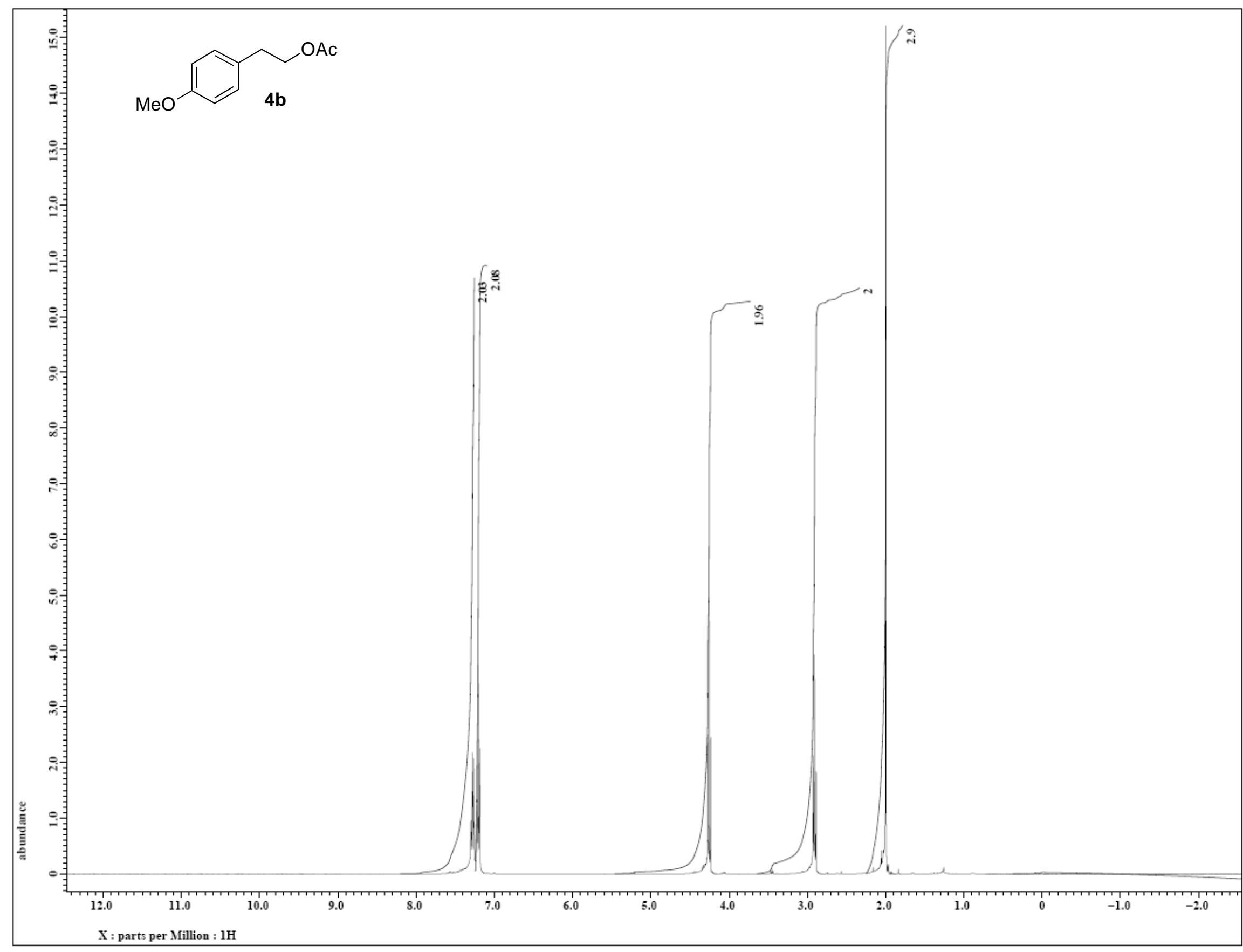




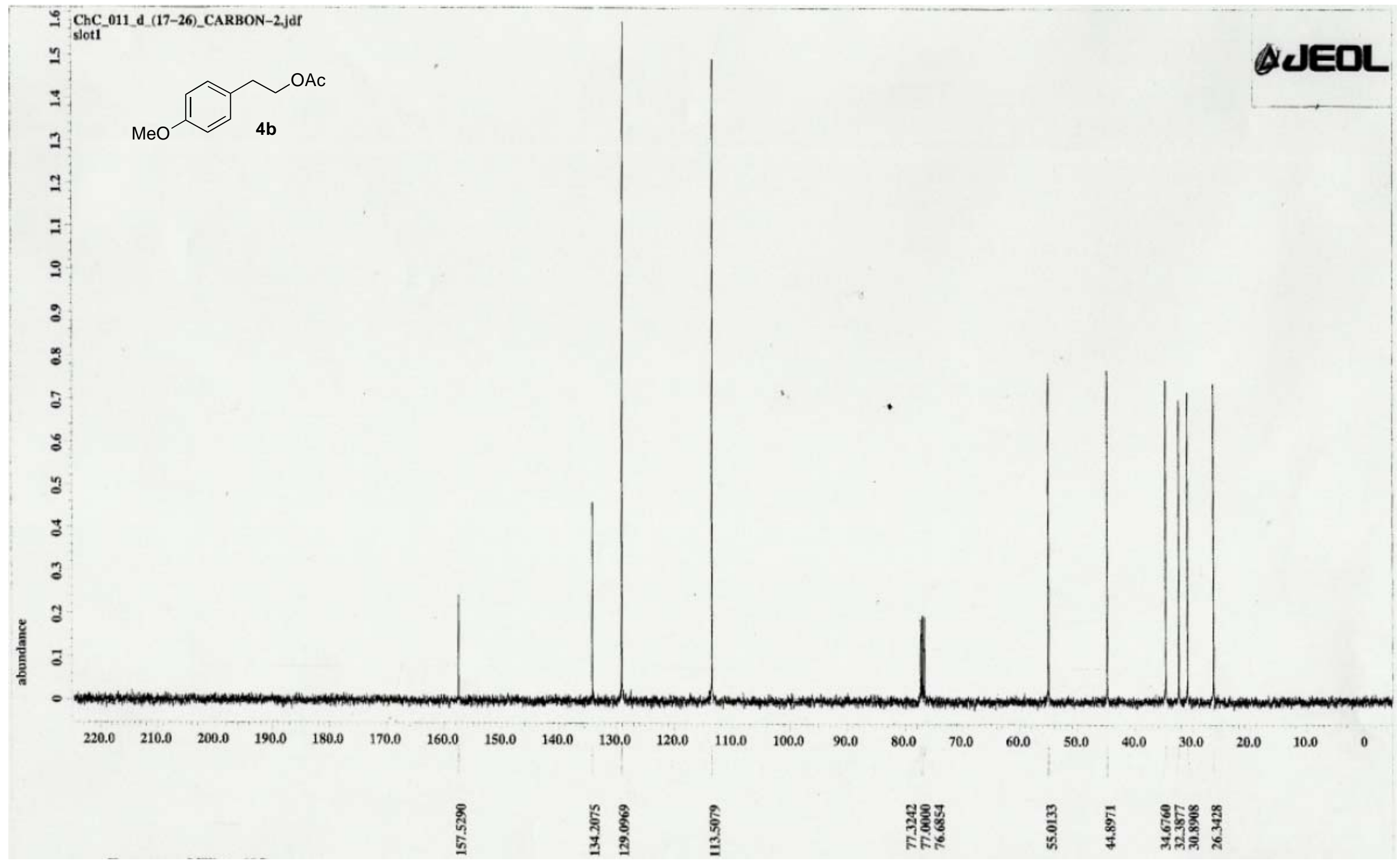




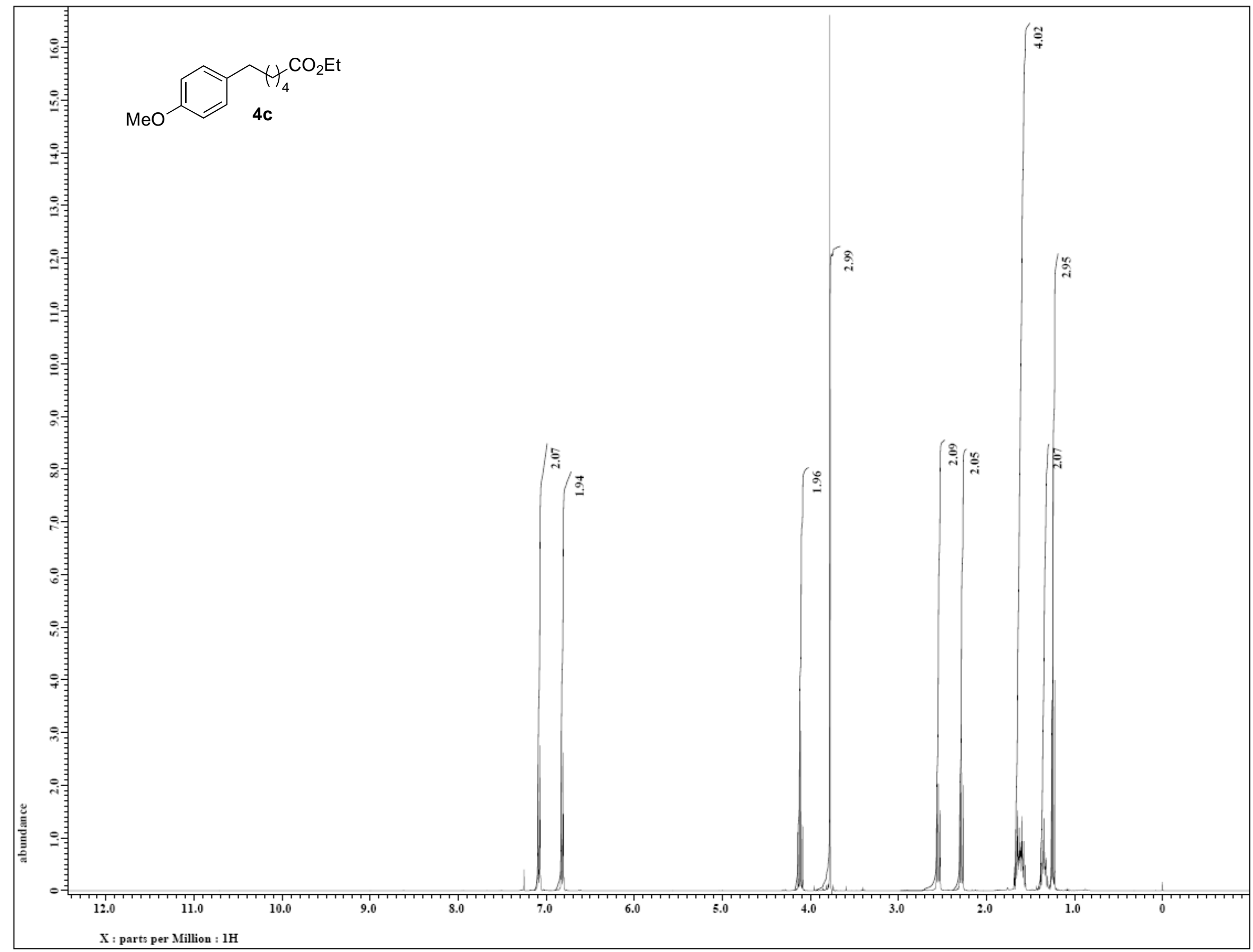




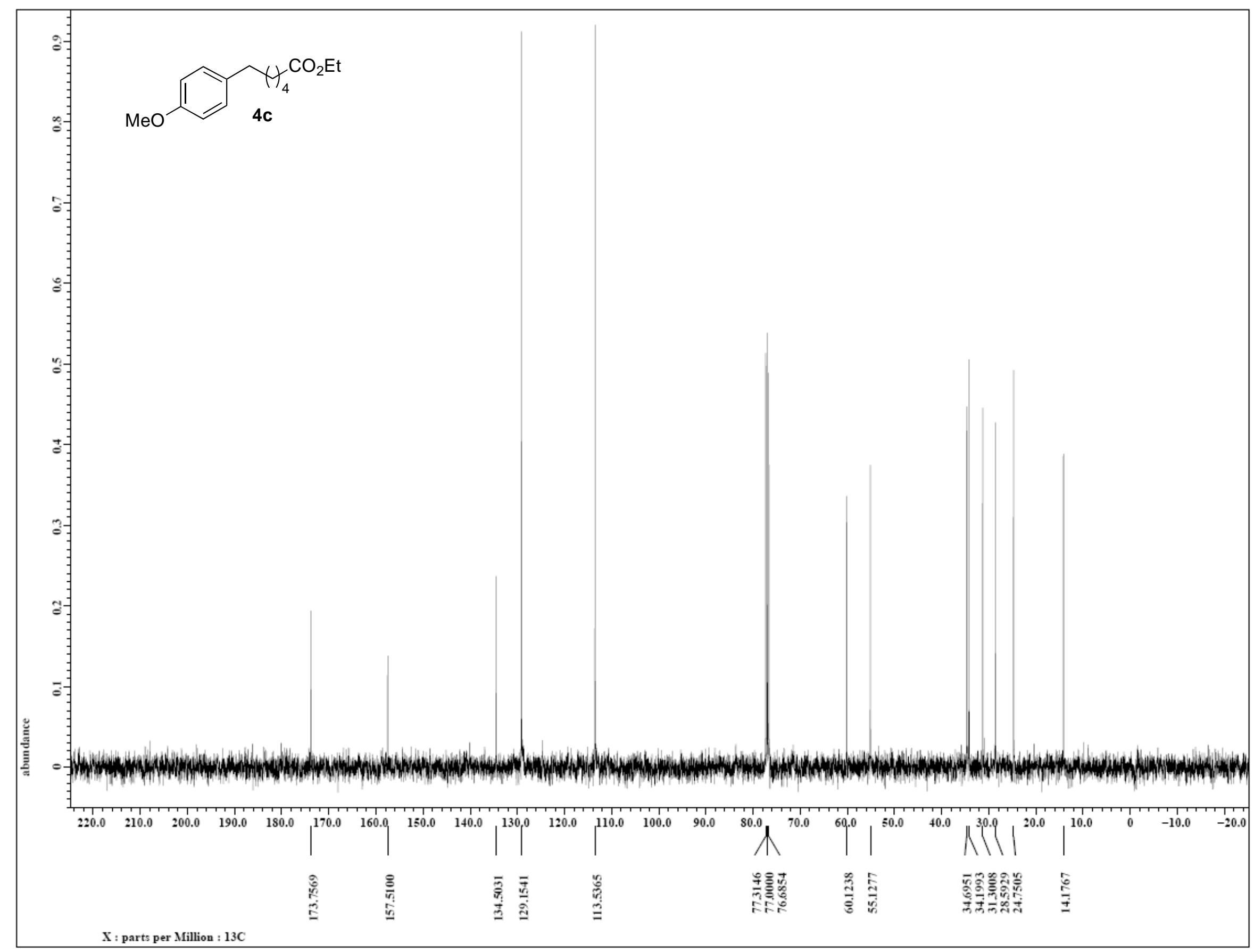




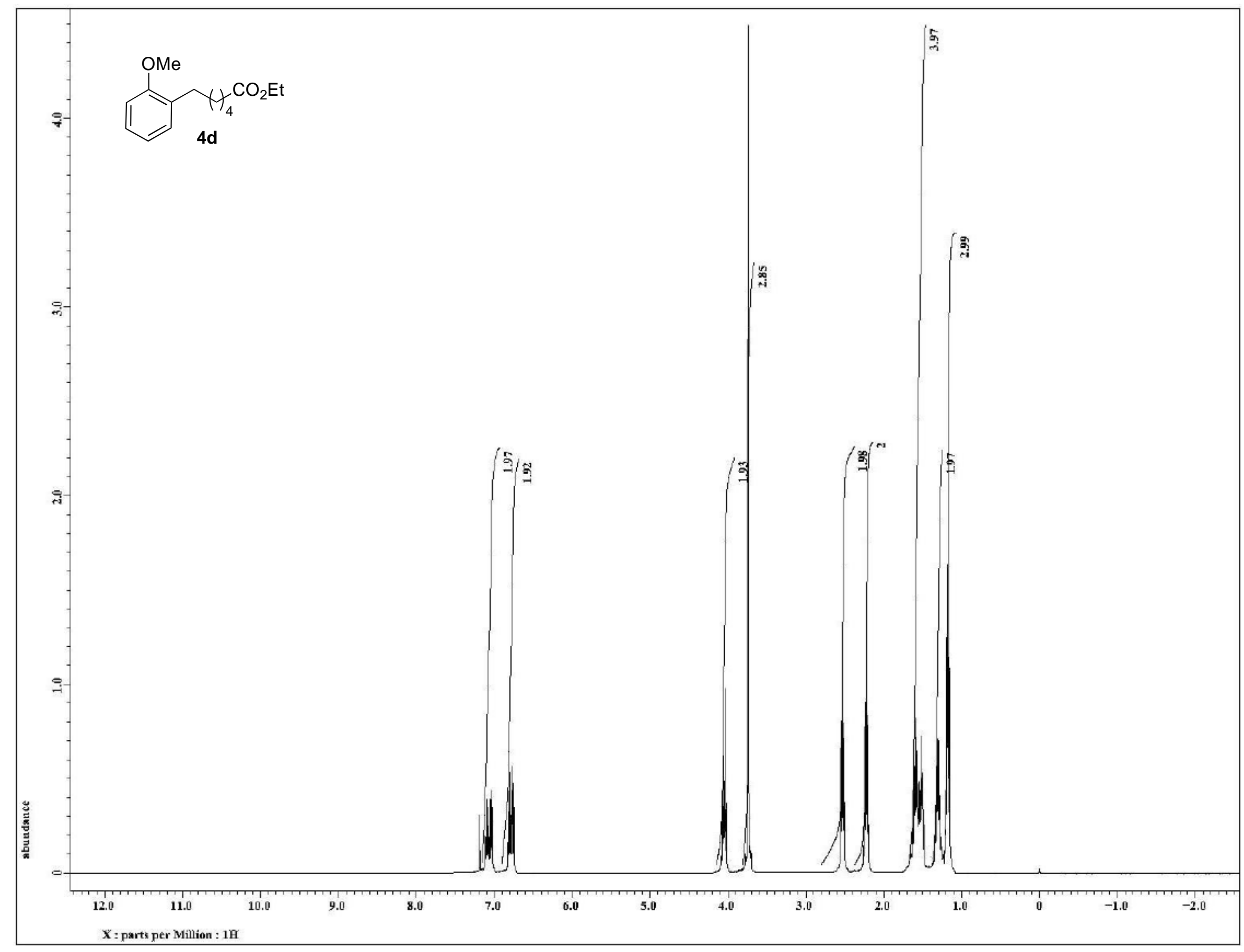




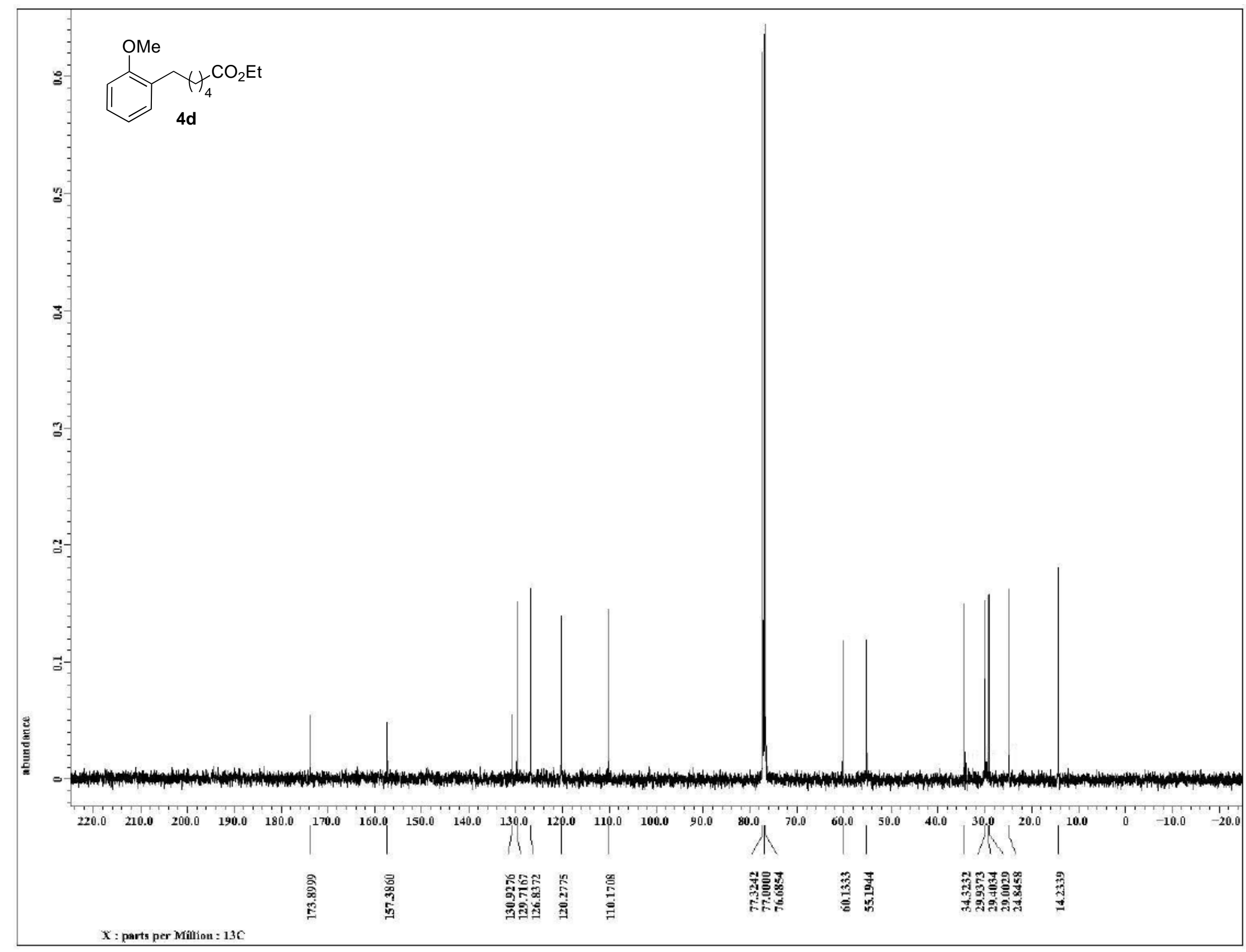




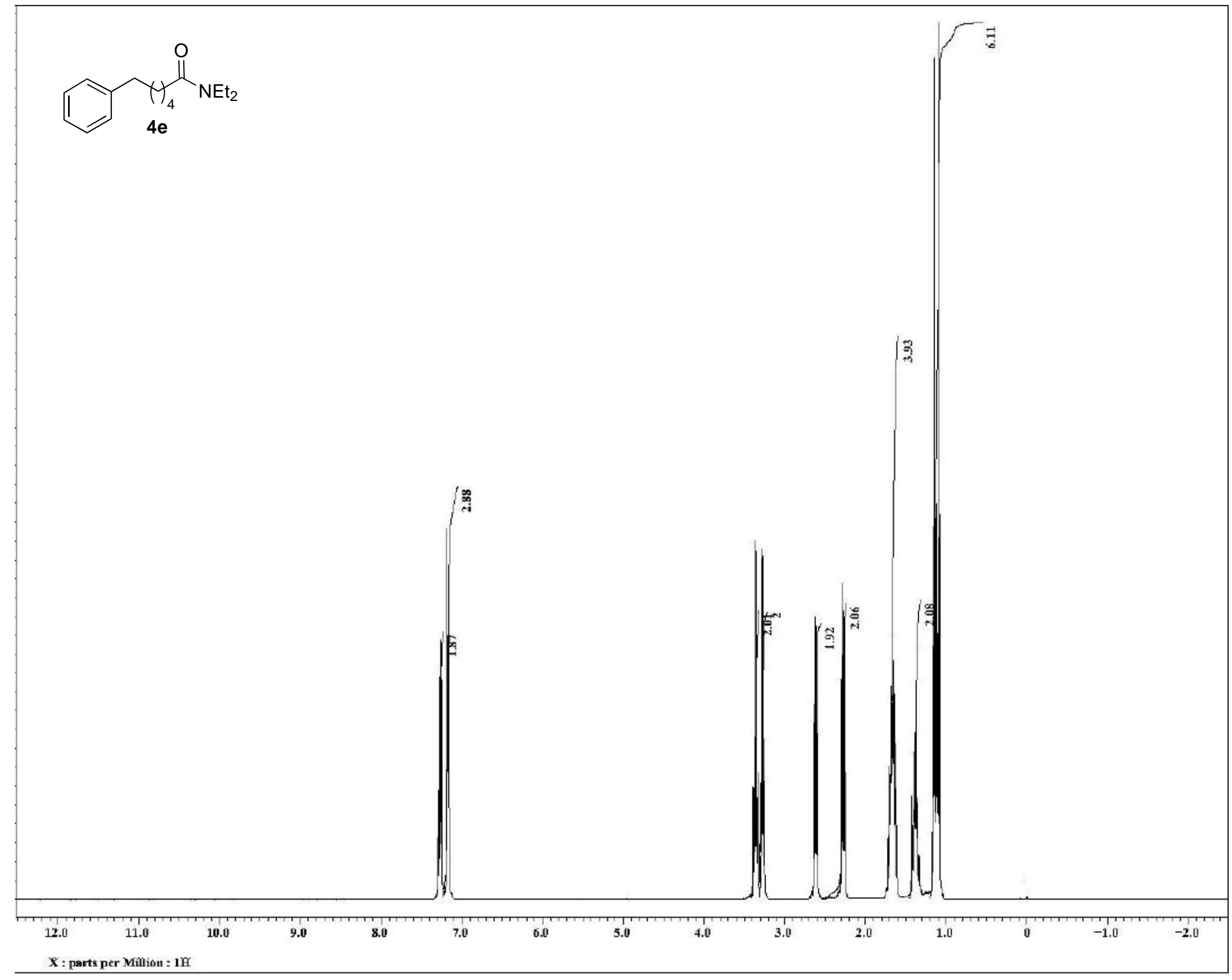




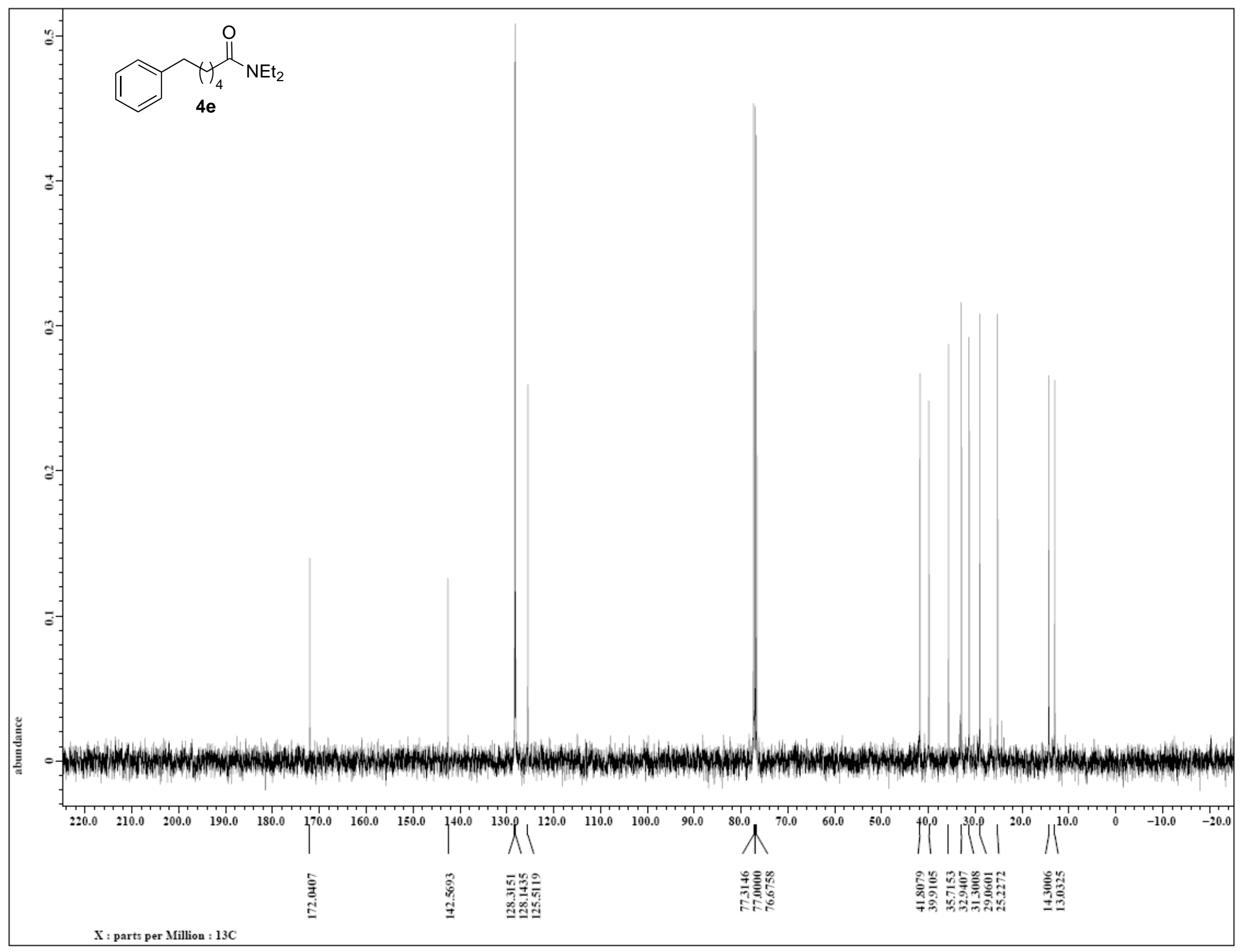




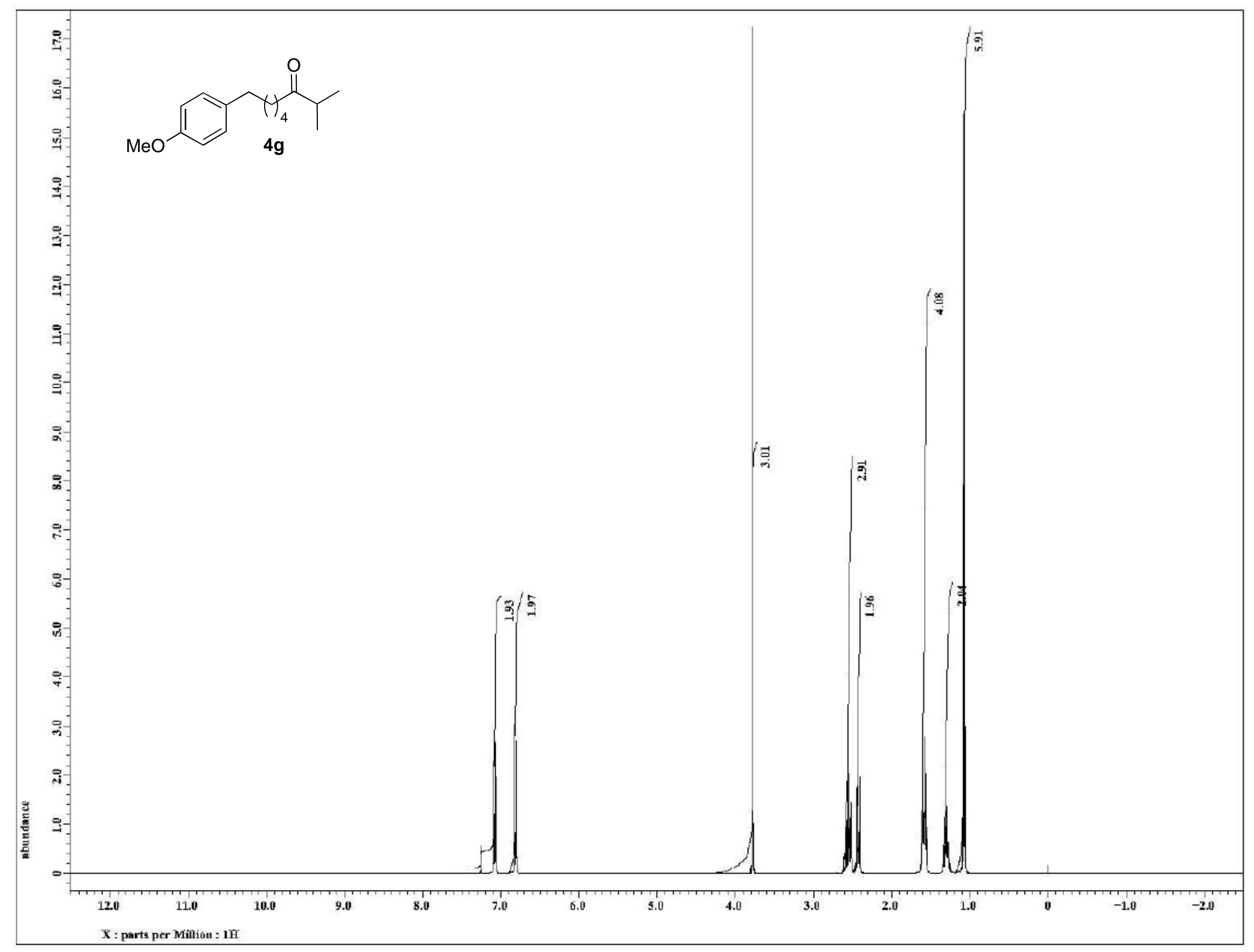




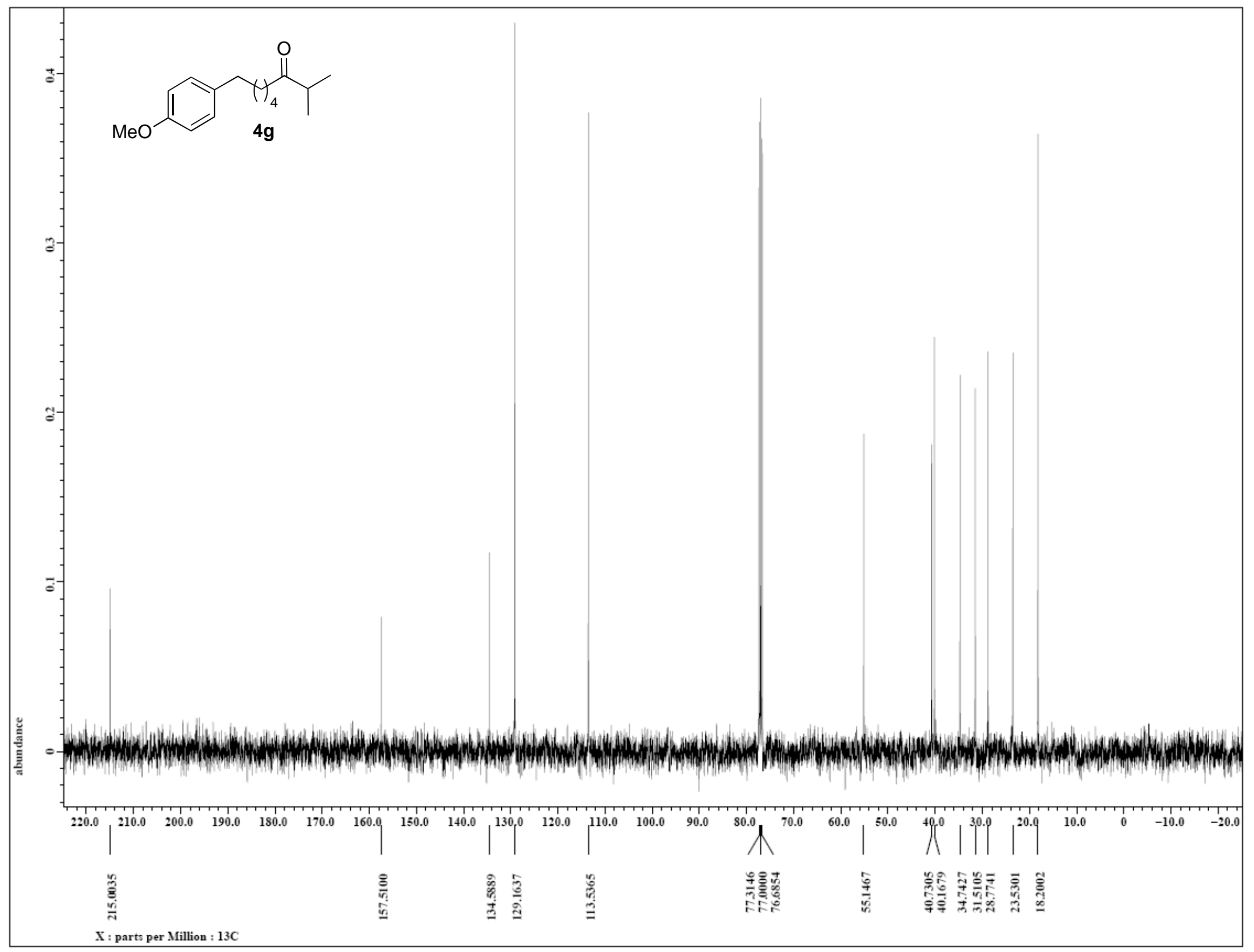




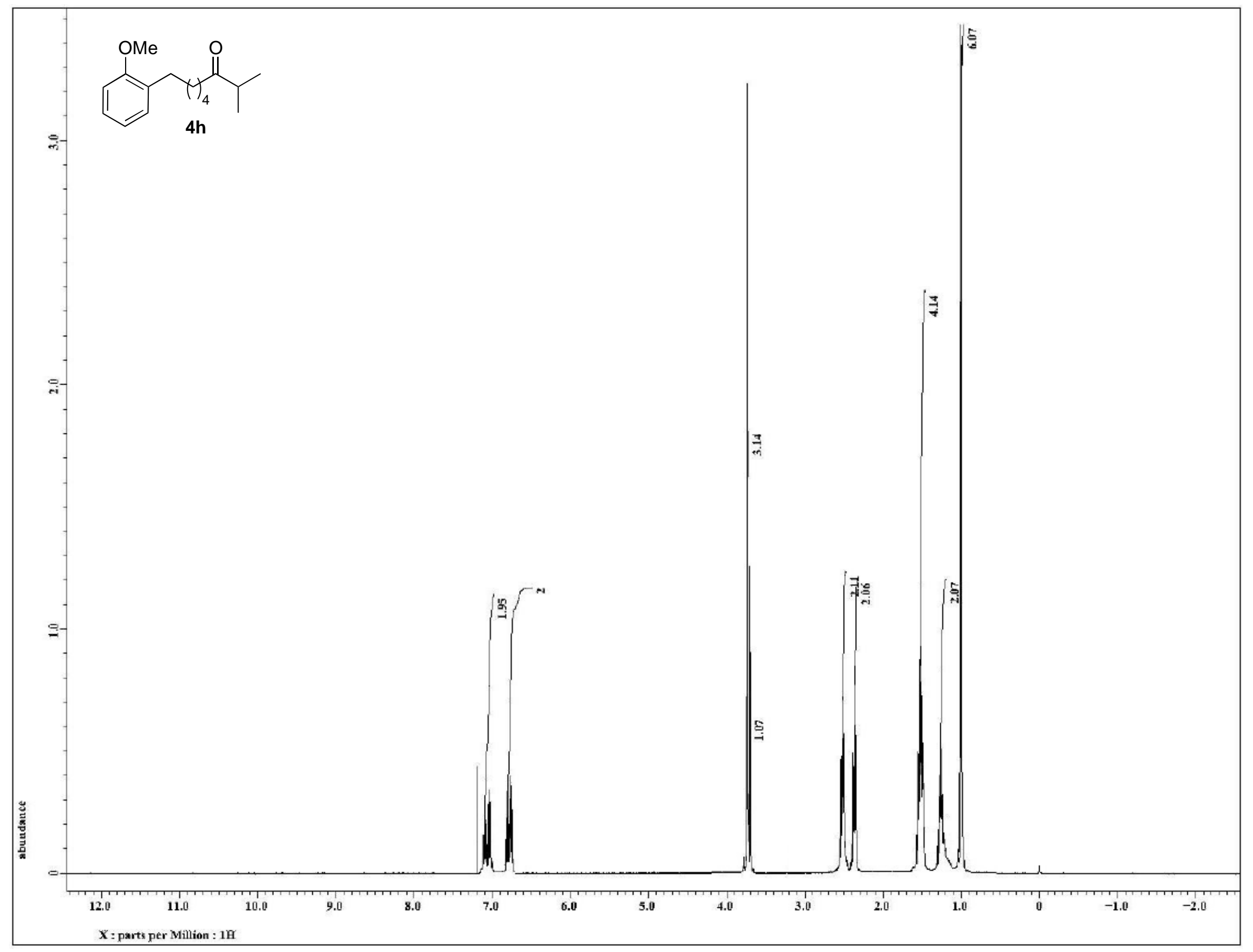




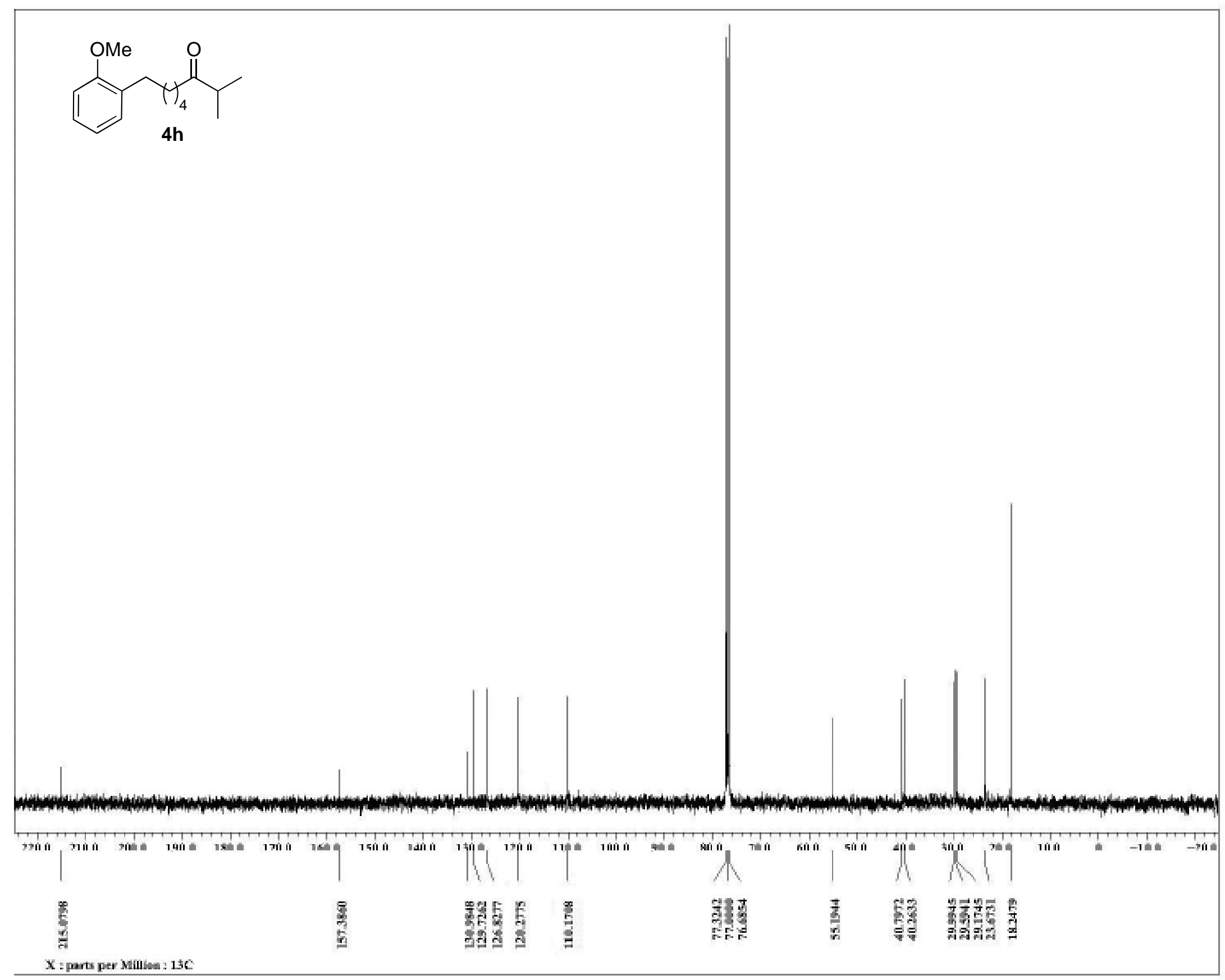

